\title{
Regularity of ghosts in tensor tomography
}

\author{
Vladimir Sharafutdinov* \\ Sobolev Institute of Mathematics \\ 4 Koptjug Avenue \\ Novosibirsk, 630090, Russia \\ sharaf@math.nsc.ru
}

\author{
Michal Skokan ${ }^{\dagger}$ \\ Department of Mathematics \\ University of Washington \\ Seattle, WA 98195, USA \\ skokan@math.washington.edu
}

\author{
Gunther Uhlmann \\ Department of Mathematics \\ University of Washington \\ Seattle, WA 98195, USA \\ gunther@math.washington.edu
}

\begin{abstract}
We study on a compact Riemannian manifold with boundary the ray transform $I$ which integrates symmetric tensor fields over geodesics. A tensor field is said to be a nontrivial ghost if it is in the kernel of $I$ and is $L^{2}$-orthogonal to all potential fields. We prove that a nontrivial ghost is smooth in the case of a simple metric. This implies that the the wave front set of the solenoidal part of a field $f$ can be recovered from the ray transform If. We give an explicit procedure for recovering the wave front set.
\end{abstract}

Key words: Integral geometry, Ray transform, Pseudodifferential operators AMS Subject Classification:

\section{Introduction}

The term ghosts is used in tomography for objects that give no contribution to the projection data. In classical scalar tomography ghosts occur only due to the discretization of a problem or in problems with incomplete data. In both the cases, ghosts turn out to be very singular functions that have either rapid oscillation or many jumps. The situation is quite different in tensor tomography when we have to recover several unknown functions from the same projection data. For example, the typical problem of Doppler tomography is described as follows. One attempts to recover a vector field $f$ on a convex bounded

*Partly supported by NSF grant DMS-0404096 and by RFBR Grant 05-01-00611.

${ }^{\dagger}$ The work was mostly done when the second author visited the Sobolev Institute of Mathematics. The author is grateful to the whole staff of the Institute for their hospitality.

${ }^{\ddagger}$ Partly supported by NSF and a John Simon Guggenheim fellowship. 
domain $M \subset \mathbf{R}^{3}$ given the work of $f$ over every straight-line segment with endpoints in $\partial M$. There is a large space of ghosts in the latter problem. Indeed, if $\varphi$ is a function on $M$ vanishing on the boundary, $\left.\varphi\right|_{\partial M}=0$, then the potential vector field $f=\nabla \varphi$ has zero work over any path with endpoints in $\partial M$. It can be easily shown that the space of ghosts coincides with the space of potential fields in the latter problem. The question is much more delicate for the following generalization of the problem which is still of great applied interest. Assume $M$ to contain some medium that refracts ultra-sound waves. The index of refraction can be often modeled by a Riemannian metric, $g$, and the ultrasound rays propagate along the geodesics of $g$. The Doppler tomography problem in this setting can be posed as follows. Can one recover a vector field $f$ in $M$ given the work of $f$ over all geodesic segments of a given metric $g$ with endpoints in $\partial M$ ? The definition of a potential field can be given in the latter setting with an obvious modification. The case of integrating tensor fields of rank higher than one appears also in several situations. For instance the case of tensor fields of rank two corresponds to the linearization of the boundary rigidity problem; see Chapter 1 of [7]. The case of tensor fields of rank four describes the perturbation of travel times of compressional waves propagating in slightly anisotropic elastic media; see Chapter 7 of [7]. We can also define potential fields in this more general setting and they are ghosts for the integration of tensor fields along geodesics. The identification of the space of ghosts with the space of potential fields is known under some assumptions on the curvature tensor of $g$, but the question is open in the case of a general metric $g$. Let us refer to potential fields as trivial ghosts and use the term nontrivial ghosts for ghosts that are $L^{2}$-orthogonal to all potential fields. In the present article we prove that, under some weak assumptions on the metric $g$, any nontrivial ghost is $C^{\infty}$-smooth. The result is obtained for symmetric tensor fields of arbitrary rank $m$, the Doppler tomography problem corresponds to the case of $m=1$. We emphasize that, until now, no example is known where the space of nontrivial ghosts is nonzero.

We describe below the problem more precisely.

Given a Riemannian manifold $(M, g)$, we denote by $C^{\infty}\left(S^{m} \tau_{M}^{*}\right)$ the space of smooth covariant symmetric tensor fields of rank $m$ on $M$. The first order differential operator

$$
d=\sigma \nabla: C^{\infty}\left(S^{m-1} \tau_{M}^{*}\right) \rightarrow C^{\infty}\left(S^{m} \tau_{M}^{*}\right)
$$

is called the inner derivative. Here $\nabla$ is the covariant derivative with respect to the metric $g$ and $\sigma$ denotes the symmetrization.

We are going to integrate symmetric tensor fields over geodesics joining boundary points. First of all we will distinguish some class of Riemannian manifolds for which the integration leads to a good operator.

A compact Riemannian manifold $(M, g)$ with boundary is called a convex non-trapping manifold (CNTM briefly), if it satisfies two conditions: (1) the boundary $\partial M$ is strictly convex, i.e., the second fundamental form of the boundary is positive definite at every boundary point; (2) for every point $x \in M$ and every vector $0 \neq \xi \in T_{x} M$, the maximal geodesic $\gamma_{x, \xi}(t)$ satisfying the initial conditions $\gamma_{x, \xi}(0)=x$ and $\dot{\gamma}_{x, \xi}(0)=\xi$ is defined on a finite segment $\left[\tau_{-}(x, \xi), \tau_{+}(x, \xi)\right]$. We recall that a geodesic $\gamma:[a, b] \rightarrow M$ is maximal if it cannot be extended to a segment $\left[a-\varepsilon_{1}, b+\varepsilon_{2}\right]$, where $\varepsilon_{i} \geq 0$ and $\varepsilon_{1}+\varepsilon_{2}>0$. The second condition is equivalent to all geodesics having finite length in $M$.

Remark. In [7], the term CDRM (compact dissipative Riemannian manifold) was used instead of CNTM. 
An important subclass of CNTMs are the simple manifolds. A compact Riemannian manifold $(M, g)$ is said to be simple if (1) it is simply connected, (2) the boundary is strictly convex, and (3) there are no conjugate points on any geodesic. A simple $n$ dimensional Riemannian manifold is diffeomorphic to a closed ball in $\mathbf{R}^{n}$, and any two points of the manifold are joined by a unique geodesic.

We denote by $T M=\left\{(x, \xi) \mid x \in M, \xi \in T_{x} M\right\}$ the tangent bundle of the manifold $M$, and by $\Omega M=\{(x, \xi) \in T M|| \xi \mid=1\}$ we denote the unit sphere bundle. We introduce the submanifolds of inner and outer vectors of $\Omega M$ :

$$
\partial_{ \pm} \Omega M=\{(x, \xi) \in \Omega M \mid x \in \partial M, \pm\langle\xi, \nu(x)\rangle \geq 0\},
$$

where $\nu$ is the unit outer normal to the boundary. Note that $\partial_{+} \Omega M$ and $\partial_{-} \Omega M$ are compact manifolds with the same boundary $\Omega(\partial M)$, and $\partial \Omega M=\partial_{+} \Omega M \cup \partial_{-} \Omega M$. It is convenient to parameterize the set of maximal oriented geodesics by points of the manifold $\partial_{+} \Omega M$. Namely, for $(x, \xi) \in \partial_{+} \Omega M$, we denote by $\gamma_{x, \xi}:\left[\tau_{-}(x, \xi), 0\right] \rightarrow M$ the maximal geodesic satisfying the initial conditions $\gamma_{x, \xi}(0)=x$ and $\dot{\gamma}_{x, \xi}(0)=\xi$.

Let $C^{\infty}\left(\partial_{+} \Omega M\right)$ be the space of smooth functions on the manifold $\partial_{+} \Omega M$. The ray transform (also called geodesic X-ray transform) on a CNTM $M$ is the linear operator

$$
I: C^{\infty}\left(S^{m} \tau_{M}^{*}\right) \rightarrow C^{\infty}\left(\partial_{+} \Omega M\right)
$$

defined by

$$
I f(x, \xi)=\int_{\tau_{-}(x, \xi)}^{0}\left\langle f\left(\gamma_{x, \xi}(t)\right), \dot{\gamma}_{x, \xi}^{m}(t)\right\rangle d t=\int_{\tau_{-}(x, \xi)}^{0} f_{i_{1} \ldots i_{m}}\left(\gamma_{x, \xi}(t)\right) \dot{\gamma}_{x, \xi}^{i_{1}}(t) \ldots \dot{\gamma}_{x, \xi}^{i_{m}}(t) d t
$$

Throughout the paper, we use the agreement: summation from 1 to $n=\operatorname{dim} M$ is assumed over upper and low indices repeated in a monomial. The right-hand side of (1.3) is a smooth function on $\partial_{+} \Omega M$ because the integration limit $\tau_{-}(x, \xi)$ is a smooth function on $\partial_{+} \Omega M$, see Lemma 4.1.1 of [7].

On a compact manifold $M$, the topological Hilbert space $H^{k}\left(S^{m} \tau_{M}^{*}\right)$ consists of rank $m$ symmetric tensor fields whose coordinates, in any local coordinate system, are locally square integrable together with partial derivatives of order $\leq k$. In a similar way we define the topological Hilbert space $H^{k}\left(\partial_{+} \Omega M\right)$ of functions on $\partial_{+} \Omega M$. (By a topological Hilbert space we mean a topological vector space whose topology can be determined by some Hilbert dot-product but the product is not fixed.) The ray transform on a CNTM is extendible to the bounded operator

$$
I: H^{k}\left(S^{m} \tau_{M}^{*}\right) \rightarrow H^{k}\left(\partial_{+} \Omega M\right)
$$

for every integer $k \geq 0$, see Theorem 4.2.1 of [7].

We denote the kernel of operator (1.4) by $Z^{k}\left(S^{m} \tau_{M}^{*}\right)$. A field $f \in H^{k}\left(S^{m} \tau_{M}^{*}\right)$ is said to be a potential field if it can be represented in the form $f=d v$ with some $v \in H^{k+1}\left(S^{m-1} \tau_{M}^{*}\right)$ satisfying the boundary condition $\left.v\right|_{\partial M}=0$. Let $P^{k}\left(S^{m} \tau_{M}^{*}\right)$ be the subspace, of $H^{k}\left(S^{m} \tau_{m}^{*}\right)$, consisting of all potential fields. For any CNTM, we have that

$$
P^{k}\left(S^{m} \tau_{M}^{*}\right) \subset Z^{k}\left(S^{m} \tau_{M}^{*}\right) .
$$

The main question on the ray transform is the following: For what classes of CNTMs and for what values of $k$ and $m$ can the inclusion in (1.5) be replaced by equality? As 
can be easily shown, if the answer is positive for $k=k_{0}$, then it is positive for $k \geq k_{0}$. No example of a CNTM is known for which (1.5) is not equality. On the other hand, equality in (1.5) is known under some assumptions on the curvature of $g$ which imply the simplicity of $(M, g)$, see Theorem 4.3 .3 of [7] and also [10], [5], [3]. With no curvature restriction, the following result is proved in [8]:

For a simple Riemannian manifold, inclusion (1.5) is of a finite codimension for all $m$ and $k \geq 1$.

The following question was posed in [8]: Given a simple Riemannian manifold, is the codimension $c_{k, m}(M, g)$ of the inclusion (1.5) independent of $k$ ? In other words, does there exist a complement of $P^{k}\left(S^{m} \tau_{M}^{*}\right)$, in $Z^{k}\left(S^{m} \tau_{M}^{*}\right)$, consisting of smooth tensor fields? The main result of the present article is the positive answer to this question. Before formulating the result, we will introduce a couple of definitions.

We want to distinguish a subspace in $H^{k}\left(S^{m} \tau_{M}^{*}\right)$ which is the complement to the space of potential fields. The most natural candidate for such a complement is the kernel of the dual to $d$ operator.

Given a Riemannian manifold $(M, g)$, the divergence operator $\delta: C^{\infty}\left(S^{m} \tau_{M}^{*}\right) \rightarrow$ $C^{\infty}\left(S^{m-1} \tau_{M}^{*}\right)$ is defined in local coordinates by $(\delta u)_{i_{1} \ldots i_{m-1}}=g^{j k} \nabla_{k} u_{j i_{1} \ldots i_{m-1}}$. The operators $d$ and $-\delta$ are dual to each other with respect to the $L^{2}$-product

$$
(u, v)_{L^{2}}=\int_{M}\langle u(x), v(x)\rangle d V^{n}(x)
$$

on the space $L^{2}\left(S^{m} \tau_{M}^{*}\right)=H^{0}\left(S^{m} \tau_{M}^{*}\right)$. Here $d V^{n}(x)=\left[\operatorname{det}\left(g_{i j}\right)\right]^{1 / 2}\left|d x^{1} \wedge \ldots \wedge d x^{n}\right|$ is the Riemannian volume form and $\langle u, v\rangle=g^{i_{1} j_{1}} \ldots g^{i_{m} j_{m}} u_{i_{1} \ldots i_{m}} \bar{v}_{j_{1} \ldots j_{m}}$ is the pointwise scalar product of tensors. A field $f \in H^{k}\left(S^{m} \tau_{M}^{*}\right)(k \geq 0)$ is said to be a solenoidal field if $\delta f=0$. The space of solenoidal fields is the orthogonal complement of the space $P^{k}\left(S^{m} \tau_{M}^{*}\right)$ of potential fields with respect to the $L^{2}$-product. For $f \in L^{2}\left(S^{m} \tau_{M}^{*}\right), d f$ and $\delta f$ are understood in the sense of distributions.

We can now state the main result of the present article.

Theorem 1.1 Let $(M, g)$ be a simple Riemannian manifold. For any $m \geq 0$, if a tensor field $f \in L^{2}\left(S^{m} \tau_{M}^{*}\right)$ is solenoidal, $\delta f=0$, and is in the kernel of the ray transform, If $=0$, then $f$ is smooth, i.e., $f \in C^{\infty}\left(S^{m} \tau_{M}^{*}\right)$.

We remark that in the cases of $m=0$ and $m=1$ the statement is trivial because (1.5) is known to be equality in these cases, see [8]. So, the first nontrivial case is $m=2$. In the latter case, the theorem was recently proved in [1] and [11]. The analytical part of our proof follows these articles with some improvements. The algebraic part of the proof is much more complicated in the case of an arbitrary $m$.

Any tensor field $f \in H^{k}\left(S^{m} \tau_{M}^{*}\right), k \geq 0$, on a compact Riemannian manifold can be uniquely represented in the form

$$
f=\tilde{f}+d v, \quad \delta \tilde{f}=0,\left.\quad v\right|_{\partial M}=0
$$

with $\tilde{f} \in H^{k}\left(S^{m} \tau_{M}^{*}\right)$ and $v \in H^{k+1}\left(S^{m-1} \tau_{M}^{*}\right)$. See Theorem 3.3.2 of [7] where the statement is proved for $k \geq 1$. The same proof with minor modifications works in the case of $k=0$. The fields $\tilde{f}$ and $d v$ are called respectively solenoidal and potential parts of the field $f$. 
Corollary 1.2 Let $(M, g)$ be a simple Riemannian manifold. For any $m \geq 0$, the wave front set of the solenoidal part $\tilde{f}$ of a tensor field $f \in L^{2}\left(S^{m} \tau_{M}^{*}\right)$ can be uniquely recovered from the ray transform If.

By the wave front set of a symmetric tensor field $f$, we mean the wave front set of the function $F(x, \xi)=f_{i_{1} \ldots i_{m}}(x) \xi^{i_{1}} \ldots \xi^{i_{m}}$ on the manifold $T M$. So, the wave front set is a closed conic subset of $T^{*}(T M) \backslash\{0\}$. Equivalently, in local coordinates, this means the family of wave front sets of all coordinates of $f$. Indeed, let $f_{1}$ and $f_{2}$ be two tensor fields such that $I f_{1}=I f_{2}$. Let $\tilde{f}_{1}$ and $\tilde{f}_{2}$ be the solenoidal parts of $f_{1}$ and $f_{2}$ respectively. By Theorem 1.1, $\tilde{f}_{1}-\tilde{f}_{2}$ is a smooth tensor field. This means that $W F\left(\tilde{f}_{1}\right)=W F\left(\tilde{f}_{2}\right)$. In Section 3, we will give an effective procedure of recovering $W F\left(\left.\tilde{f}\right|_{M \backslash \partial M}\right)$.

The article is organized as follows. In Section 2, we consider the dual $I^{*}$ of the ray transform $I$, prove that $I^{*} I$ is a pseudodifferential operator of order -1 , and calculate the principal symbol of the operator. In Section 3, we construct a parametrix for $I^{*} I$ on the space of solenoidal tensor fields. Our construction of the parametrix has many features in common with that of [11] but is done in more invariant terms. In Section 4, we recall some known facts about the transmission condition which are presented in the form that we need. In Section 5, we prove the interior regularity of a field $f$ as in Theorem 1.1 and reduce the question of the boundary regularity to proving ellipticity of some operator of the form $E-\mathcal{A}$ on $\partial M$, where $E$ is the identity. We calculate the principal symbol $A$ of $\mathcal{A}$ in Section 6. The rest of the article is of a pure algebraic nature. After developing some tensor machinery in Section 7, we find invariant subspaces of the operator $A$ in Section 8. The question is thus reduced to checking nonsingularity of some matrices $E-A^{(l)}(0 \leq l \leq m)$ whose elements are expressed in terms of binomial coefficients. Section 9 contains some preliminaries on binomial coefficients. Finally, in Section 10, we calculate the spectrum of the matrix $A^{(l)}$ and prove that $E-A^{(l)}$ is a nonsingular matrix.

The statement of Theorem 10.1 on spectra of matrices $A^{(l)}$ was found on the base of many numerical experiments with these matrices. The authors are grateful to Yuri Krivtsov for his help with the numerical calculations.

\section{$2 \quad$ Symbol of $I^{*} I$}

Given a Riemannian manifold $(M, g)$, we denote by $T_{x} M$ the tangent space at $x \in M$, and by $T_{x}^{*} M$, the dual space. The metric $g$ defines the canonical isomorphism between $T_{x} M$ and $T_{x}^{*} M$ which is expressed in coordinates by the well-known rule of raising and lowering indices of a vector: $\xi_{i}=g_{i j} \xi^{j}$ and $\xi^{i}=g^{i j} \xi_{j}$. The same rule applies to tensors. Let $S^{m} \tau_{M}^{*}$ be the complex vector bundle of covariant symmetric tensors of rank $m$ over $M$. The fiber of the bundle over a point $x \in M$ is denoted by $S^{m}\left(T_{x}^{*} M\right)$. The scalar product on $S^{m}\left(T_{x}^{*} M\right)$ is defined in coordinates by $\langle u, v\rangle=u^{i_{1} \ldots i_{m}} \overline{v_{i_{1} \ldots i_{m}}}$, the result is independent of the choice of coordinates. The symmetric product $u v=\sigma(u \otimes v)$, where $\sigma$ is the symmetrization, turns $\oplus_{m=0}^{\infty} S^{m}\left(T_{x}^{*} M\right)$ into a commutative graded algebra. For $\xi \in T_{x}^{*} M$, we denote by $i_{\xi}: S^{m}\left(T_{x}^{*} M\right) \rightarrow S^{m+1}\left(T_{x}^{*} M\right)$ the operator of symmetric multiplication by $\xi$ and by $j_{\xi}: S^{m}\left(T_{x}^{*} M\right) \rightarrow S^{m-1}\left(T_{x}^{*} M\right)$, the dual of $i_{\xi}$.

For an $n$-dimensional Riemannian manifold $(M, g)$ and a point $x \in M$, by $\Omega_{x} M=$ $\left\{\left.\xi \in T_{x} M|| \xi\right|^{2}=g_{i j}(x) \xi^{i} \xi^{j}=1\right\}$ we denote the unit sphere in the tangent space $T_{x} M$. The Riemannian scalar product on $T_{x} M$ induces the volume form on $\Omega_{x} M$ which is denoted by $d \omega_{x}(\xi)$. We introduce the volume form $d \Sigma^{2 n-1}$ on the manifold $\Omega M$ by 
$d \Sigma^{2 n-1}(x, \xi)=\left|d \omega_{x}(\xi) \wedge d V^{n}(x)\right|$, where $d V^{n}$ is the Riemannian volume form on $M$. By the well known Liouville theorem, the form $d \Sigma^{2 n-1}$ is preserved by the geodesic flow. The corresponding volume form on the boundary $\partial \Omega M=\{(x, \xi) \in \Omega M \mid x \in \partial M\}$ is given by $d \Sigma^{2 n-2}=\left|d \omega_{x}(\xi) \wedge d V^{n-1}\right|$, where $d V^{n-1}$ is the volume form of $\partial M$.

Let $(M, g)$ be a convex non-trapping Riemannian manifold. Introduce the space $L^{2}\left(\partial_{+} \Omega M\right)$ by

$$
(\varphi, \psi)_{L^{2}\left(\partial_{+} \Omega M\right)}=\int_{\partial_{+} \Omega M}\langle\xi, \nu(x)\rangle \varphi(x, \xi) \overline{\psi(x, \xi)} d \Sigma^{2 n-2}(x, \xi) .
$$

As has been mentioned in Section 1, the ray transform

$$
I: L^{2}\left(S^{m} \tau_{M}^{*}\right) \rightarrow L^{2}\left(\partial_{+} \Omega M\right)
$$

is a bounded operator. Now, we calculate its adjoint $I^{*}$.

For $f \in L^{2}\left(S^{m} \tau_{M}^{*}\right)$ and $\psi \in L^{2}\left(\partial_{+} \Omega M\right)$,

$$
\begin{gathered}
(I f, \psi)_{L^{2}\left(\partial_{+} \Omega M\right)}=\int_{\partial_{+} \Omega M}\langle\xi, \nu(x)\rangle \operatorname{If}(x, \xi) \overline{\psi(x, \xi)} d \Sigma^{2 n-2}(x, \xi)= \\
=\int_{\partial_{+} \Omega M}\langle\xi, \nu(x)\rangle\left[\int_{\tau_{-}(x, \xi)}^{0} f_{i_{1} \ldots i_{m}}\left(\gamma_{x, \xi}(t)\right) \dot{\gamma}_{x, \xi}^{i_{1}}(t) \ldots \dot{\gamma}_{x, \xi}^{i_{m}}(t) d t\right] \overline{\psi(x, \xi)} d \Sigma^{2 n-2}(x, \xi) .
\end{gathered}
$$

Introduce the domain

$$
G=\left\{(x, \xi ; t) \mid(x, \xi) \in \partial_{+} \Omega M, \tau_{-}(x, \xi) \leq t \leq 0\right\} \subset \partial_{+} \Omega M \times \mathbf{R}
$$

and map

$$
\lambda: G \rightarrow \Omega M, \quad \lambda(x, \xi ; t)=\left(\gamma_{x, \xi}(t), \dot{\gamma}_{x, \xi}(t)\right) .
$$

It is a diffeomorphism at least in the interior of $G$. It can be easily checked using Liouville's theorem that

$$
\lambda^{*}\left(d \Sigma^{2 n-1}\right)=\langle\xi, \nu\rangle d t \wedge d \Sigma^{2 n-2} .
$$

Changing variables with the help of $\lambda$ and using the latter equality, we transform (2.1) to the following:

$$
(I f, \psi)_{L^{2}\left(\partial_{+} \Omega M\right)}=\int_{\Omega M} f_{i_{1} \ldots i_{m}}(x) \xi^{i_{1}} \ldots \xi^{i_{m}} \overline{\psi\left(\gamma_{x, \xi}\left(\tau_{+}(x, \xi)\right), \dot{\gamma}_{x, \xi}\left(\tau_{+}(x, \xi)\right)\right)} d \Sigma^{2 n-1}(x, \xi) .
$$

If we denote by $\tilde{\psi}$ the extension of the function $\psi$ from $\partial_{+} \Omega M$ to $\Omega M$ such that the function $\tilde{\psi}$ is constant on every orbit of the geodesic flow, then $\tilde{\psi}(x, \xi)=\psi\left(\gamma_{x, \xi}\left(\tau_{+}(x, \xi)\right), \dot{\gamma}_{x, \xi}\left(\tau_{+}(x, \xi)\right)\right)$, and the previous formula can be written as follows:

$$
(I f, \psi)_{L^{2}\left(\partial_{+} \Omega M\right)}=\int_{M} f_{i_{1} \ldots i_{m}}(x)\left[\int_{\Omega_{x} M} \xi^{i_{1}} \ldots \xi^{i_{m}} \bar{\psi} \tilde{\psi}(x, \xi) d \omega_{x}(\xi)\right] d V^{n}(x)=\left(f, I^{*} \psi\right)_{L^{2}\left(S^{m} \tau_{M}^{*}\right)},
$$

where

$$
\left(I^{*} \psi\right)^{i_{1} \ldots i_{m}}(x)=\int_{\Omega_{x} M} \xi^{i_{1}} \ldots \xi^{i_{m}} \tilde{\psi}(x, \xi) d \omega_{x}(\xi)
$$


Now, we calculate the composition $I^{*} I$.

$$
\begin{gathered}
\left(I^{*} I f\right)^{i_{1} \ldots i_{m}}(x)=\int_{\Omega_{x} M} \xi^{i_{1}} \ldots \xi^{i_{m}} \widetilde{I f}(x, \xi) d \omega_{x}(\xi) \\
=\int_{\Omega_{x} M} \xi^{i_{1}} \ldots \xi^{i_{m}} \int_{\tau_{-}(x, \xi)}^{\tau_{+}(x, \xi)} f_{j_{1} \ldots j_{m}}\left(\gamma_{x, \xi}(t)\right) \dot{\gamma}_{x, \xi}^{j_{1}}(t) \ldots \dot{\gamma}_{x, \xi}^{j_{m}}(t) d t d \omega_{x}(\xi) \\
=\int_{\Omega_{x} M} \xi^{i_{1}} \ldots \xi^{i_{m}} \int_{0}^{\tau_{+}(x, \xi)} f_{j_{1} \ldots j_{m}}\left(\gamma_{x, \xi}(t)\right) \dot{\gamma}_{x, \xi}^{j_{1}}(t) \ldots \dot{\gamma}_{x, \xi}^{j_{m}}(t) d t d \omega_{x}(\xi) \\
+\int_{\Omega_{x} M} \xi^{i_{1}} \ldots \xi^{i_{m}} \int_{\tau_{-}(x, \xi)}^{0} f_{j_{1} \ldots j_{m}}\left(\gamma_{x, \xi}(t)\right) \dot{\gamma}_{x, \xi}^{j_{1}}(t) \ldots \dot{\gamma}_{x, \xi}^{j_{m}}(t) d t d \omega_{x}(\xi) .
\end{gathered}
$$

Two integrals on the right-hand side coincide as is easily seen using the change $t=$ $-s, \xi=-\eta$. Therefore

$$
\left(I^{*} I f\right)^{i_{1} \ldots i_{m}}(x)=2 \int_{\Omega_{x} M} \xi^{i_{1}} \ldots \xi^{i_{m}} \int_{0}^{\tau_{+}(x, \xi)} f_{j_{1} \ldots j_{m}}\left(\gamma_{x, \xi}(t)\right) \dot{\gamma}_{x, \xi}^{j_{1}}(t) \ldots \dot{\gamma}_{x, \xi}^{j_{m}}(t) d t d \omega_{x}(\xi) .
$$

We change integration variables in (2.3) as follows. For a fixed point $x \in M$, we consider $(\xi, t)$ as polar coordinates in $T_{x} M$ and put $v=t \xi$. Then $d v=|v|^{n-1}\left|d t \wedge d \omega_{x}(w)\right|$ and $\dot{\gamma}_{x, \xi}(t)=J_{\exp _{x} v}^{x} v /|v|$, where $d v$ is the volume form on $T_{x} M$ and $J_{\exp _{x} v}^{x}: T_{x} M \rightarrow$ $T_{\exp _{x} v} M$ is the parallel transport along the geodesic $t \mapsto \exp _{x} t v$. After the change of variables, (2.3) takes the form

$$
\left(I^{*} I f\right)^{i_{1} \ldots i_{m}}(x)=2 \int_{T_{x} M} \frac{v^{i_{1}} \ldots v^{i_{m}}}{|v|^{n+2 m-1}} f_{j_{1} \ldots j_{m}}\left(\exp _{x} v\right)\left(J_{\exp _{x} v}^{x} v\right)^{j_{1}} \ldots\left(J_{\exp _{x} v}^{x} v\right)^{j_{m}} d v .
$$

The integration is taken over such $v \in T_{x} M$ that $\exp _{x} v$ is defined. We make below the convention that an integrand depending on $\exp _{x} v$ is assumed to be zero for the $v$ such that $\exp _{x} v$ is not defined. With this agreement in mind, we can assume the integration to be taken over whole of $T_{x} M$ in (2.4) and similar integrals below.

The parallel transport is defined on tensors as well as on vectors and agrees with the inner product, i.e.,

$$
f_{j_{1} \ldots j_{m}}\left(\exp _{x} v\right)\left(J_{\exp _{x} v}^{x} v\right)^{j_{1}} \ldots\left(J_{\exp _{x} v}^{x} v\right)^{j_{m}}=\left(J_{x}^{\exp _{x} v} f\left(\exp _{x} v\right)\right)_{j_{1} \ldots j_{m}} v^{j_{1}} \ldots v^{j_{m}} .
$$

Therefore (2.4) can be rewritten as follows:

$$
\left(I^{*} I f\right)^{i_{1} \ldots i_{m}}(x)=2 \int_{T_{x} M} \frac{v^{i_{1}} \ldots v^{i_{m}} v^{j_{1}} \ldots v^{j_{m}}}{|v|^{n+2 m-1}}\left(J_{x}^{\exp _{x} v} f\left(\exp _{x} v\right)\right)_{j_{1} \ldots j_{m}} d v
$$

We define the Fourier transform on $T_{x} M$ as the linear operator $F: \mathcal{S}^{\prime}\left(T_{x} M\right) \rightarrow$ $\mathcal{S}^{\prime}\left(T_{x}^{*} M\right)$ on the space of temporary distributions. Introduce the notation

$$
\lambda^{i_{1} \ldots i_{m} j_{1} \ldots j_{m}}(x, \xi)=2 \bar{F}_{v \rightarrow \xi}\left[\frac{v^{i_{1}} \ldots v^{i_{m}} v^{j_{1}} \ldots v^{j_{m}}}{|v|^{n+2 m-1}}\right]=2 F_{v \rightarrow \xi}\left[\frac{v^{i_{1}} \ldots v^{i_{m}} v^{j_{1}} \ldots v^{j_{m}}}{|v|^{n+2 m-1}}\right] .
$$


The latter equality holds because $\bar{F}$ is applied to an even function. By the inversion formula for the Fourier transform,

$$
2 \frac{v^{i_{1}} \ldots v^{i_{m}} v^{j_{1}} \ldots v^{j_{m}}}{|v|^{n+2 m-1}}=(2 \pi)^{-n} \int_{T_{x}^{*} M} e^{-i\langle v, \xi\rangle} \lambda^{i_{1} \ldots i_{m} j_{1} \ldots j_{m}}(x, \xi) d \xi .
$$

We substitute the latter expression into (2.5)

$$
\left(I^{*} I f\right)^{i_{1} \ldots i_{m}}(x)=(2 \pi)^{-n} \int_{T_{x} M} \int_{T_{x}^{*} M} e^{-i\langle v, \xi\rangle} \lambda^{i_{1} \ldots i_{m} j_{1} \ldots j_{m}}(x, \xi)\left(J_{x}^{\exp _{x} v} f\left(\exp _{x} v\right)\right)_{j_{1} \ldots j_{m}} d \xi d v
$$

By formulas (2.11.4)-(2.11.5) and Lemma 2.11.1 of [7],

$$
\begin{gathered}
\lambda^{i_{1} \ldots i_{m} j_{1} \ldots j_{m}}(x, \xi)=(-1)^{m} \frac{\pi^{n / 2} \Gamma(-m+1 / 2)}{2^{2 m-2} \Gamma(m+n / 2-1 / 2)} \frac{\partial^{2 m}|\xi|^{2 m-1}}{\partial \xi_{i_{1}} \ldots \partial \xi_{i_{m}} \partial \xi_{j_{1}} \ldots \partial \xi_{j_{m}}}= \\
=(-1)^{m} \frac{((2 m-1) ! !)^{2} \pi^{n / 2} \Gamma(-m+1 / 2)}{2^{2 m-2} \Gamma(m+n / 2-1 / 2)}|\xi|^{-1}\left(\varepsilon^{m}(\xi)\right)^{i_{1} \ldots i_{m} j_{1} \ldots j_{m}},
\end{gathered}
$$

where $\varepsilon^{i j}(\xi)=g^{i j}-\xi^{i} \xi^{j} /|\xi|^{2}$ and $\varepsilon^{m}(\xi)$ is the $m$-th symmetric power of the tensor $\varepsilon(\xi)$. We have thus proved

Theorem 2.1 Let $(M, g)$ be a CNTM of dimension $n$. The operator

$$
I^{*} I: C^{\infty}\left(S^{m} \tau_{M}^{*}\right) \rightarrow C^{\infty}\left(S^{m} \tau_{M}^{*}\right)
$$

is given by the formula

$$
\left(I^{*} I f\right)(x)=(2 \pi)^{-n} \int_{T_{x} M} \int_{T_{x}^{*} M} e^{-i\langle v, \xi\rangle} \lambda(x, \xi)\left(J_{x}^{\exp _{x} v} f\left(\exp _{x} v\right)\right) d \xi d v
$$

where $J_{x}^{\exp _{x} v}: S^{m}\left(T_{\exp _{x} v}^{*} M\right) \rightarrow S^{m}\left(T_{x}^{*} M\right)$ is the parallel transport of tensors along the geodesic $t \mapsto \exp _{x} t v$, the linear operator $\lambda(x, \xi): S^{m}\left(T_{x}^{*} M\right) \rightarrow S^{m}\left(T_{x}^{*} M\right)$ is defined in coordinates by the formula $(\lambda(x, \xi) f)_{i_{1} \ldots i_{m}}=\lambda_{i_{1} \ldots i_{m} j_{1} \ldots j_{m}}(x, \xi) f^{j_{1} \ldots j_{m}}$ with

$$
\lambda(x, \xi)=(-1)^{m} \frac{((2 m-1) ! !)^{2} \pi^{n / 2} \Gamma(-m+1 / 2)}{2^{2 m-2} \Gamma(m+n / 2-1 / 2)}|\xi|^{-1} \varepsilon^{m}(\xi)
$$

and $\varepsilon_{i j}(\xi)=g_{i j}-\xi_{i} \xi_{j} /|\xi|^{2}$

Remark. Our proof of Theorem 2.1 is a modification of arguments of Section 2.11 of [7]. The main result of the section, formula (2.11.6), is equivalent to Theorem 2.1 in the case of the standard Euclidean metric on $\mathbf{R}^{n}$. The operator $I^{*}$ is denoted by $\mu^{m}$ in [7].

Theorem 2.1 suggests that $I^{*} I$ is a pseudodifferential operator of order -1 . But there are two main difficulties in considering $I^{*} I$ as a pseudodifferential operator. First, pseudodifferential operators are traditionally defined on manifolds with no boundary; the theory of such operators on manifolds with boundary has several additional features and complications. Pseudodifferential operators satisfying the transmission condition is one example of such a theory (see for instance chapter 5 of [2]). In fact $I^{*} I$ belongs to this 
latter class but we will not use this here. We will overcome this difficulty by embedding our compact manifold $M$ into a larger open (= noncompact with no boundary) manifold $\tilde{M}$ and considering all operators on $\tilde{M}$. The second more essential difficulty has to do with conjugate points of the metric, i.e., critical points of the exponential map $\exp _{x}$ which appears in (2.6). In the presence of such points, the singular support of $I^{*} I f$ can be larger than that of $f$; so $I^{*} I$ is not a pseudodifferential operator. We avoid the latter difficulty by assuming $g$ to be a simple metric.

Let $(\tilde{M}, g)$ be an open Riemannian manifold. It is said to be an open simple Riemannian manifold if there exists a compact simple Riemannian manifold $\left(M^{\prime}, g^{\prime}\right)$ such that $\tilde{M}$ is the interior of $M^{\prime}$, i.e., $\tilde{M}=M^{\prime} \backslash \partial M^{\prime}$, and $g=\left.g^{\prime}\right|_{\tilde{M}}$. For such a simple open manifold $(\tilde{M}, g)$, we define the ray transform as the operator

$$
I: C_{0}^{\infty}\left(S^{m} \tau_{\tilde{M}}^{*}\right) \rightarrow C^{\infty}\left(\partial_{+} \Omega M^{\prime}\right) .
$$

Any compact simple Riemannian manifold $(M, g)$ can be embedded into an open simple Riemannian manifold $(\tilde{M}, g)$ of the same dimension.

Corollary 2.2 For an open simple Riemannian manifold $(\tilde{M}, g)$ and for any integer $m \geq$ 0 , the operator

$$
I^{*} I: C_{0}^{\infty}\left(S^{m} \tau_{\tilde{M}}^{*}\right) \rightarrow C^{\infty}\left(S^{m} \tau_{\tilde{M}}^{*}\right)
$$

is the pseudodifferential operator of order -1 with the principal symbol $\lambda(x, \xi)$ defined by (2.7).

Corollary 2.2 follows from Theorem 2.1 using the arguments presented in Section 3 of [9], see the discussion of the relation between $\Psi_{\text {global }}^{m}(X, \nabla)$ and $\Psi^{m}(X)$ before Lemma 3.1 of the latter article. Actually, Theorem 2.1 implies the following stronger statement. In terms of $[9], \lambda(x, \xi)$ is the full geometric symbol of the operator $I^{*} I$. In particular, $I^{*} I$ is a pseudodifferential operator with constant coefficients because $\stackrel{h}{\nabla} \lambda(x, \xi)=0$.

Formula (2.7) implies the following important properties of the symbol $\lambda$ :

$$
\lambda(x, \xi) i_{\xi}=0, \quad j_{\xi} \lambda(x, \xi)=0 .
$$

\section{Parametrix construction}

In this section, $M$ is a simple compact Riemannian manifold which is assumed to be a compact subset of a larger open simple Riemannian manifold $\tilde{M}$. All operators are considered on $\tilde{M}$. We denote the principal symbol of a pseudodifferential operator $A$ of order $m$ by $\sigma_{m}(A)$. From the definitions of $d$ and $\delta$, we have $\sigma_{1}(d)=i i_{\xi}, \sigma_{1}(\delta)=i j_{\xi}$, where $i$ is the imaginary unit. Recall that $\delta d$ is an elliptic operator, i.e., $\sigma_{2}(\delta d)=-j_{\xi} i_{\xi}$ : $S^{m}\left(T_{x}^{*} \tilde{M}\right) \rightarrow S^{m}\left(T_{x}^{*} \tilde{M}\right)$ is an isomorphism for any $\xi \neq 0$; see Lemma 3.3 .3 of [7]. The principal symbol $\lambda(x, \xi)=\sigma_{-1}\left(I^{*} I\right)$ of the operator $I^{*} I$ will be, for the most part, denoted by $\lambda(\xi)$ because its dependence on $x$ is not relevant for us.

Theorem 3.1 Let $S$ be a parametrix of the operator $\delta d$. There exists a pseudodifferential operator $P$ of order 1 on the bundle $S^{m} \tau_{\tilde{M}}^{*}$ such that

$$
E=P I^{*} I+d S \delta+R
$$

where $E$ is the identity operator and $R$ is a smoothing operator. 
The operator $S$ is uniquely determined by formula (3.1), i.e., the following statement is valid: if an operator $S$ satisfies (3.1) for some $P$, then $S$ is a parametrix for $\delta d$. Indeed, assume (3.1) to be valid. Choose $v \in C_{0}^{\infty}\left(S^{m-1} \tau_{\tilde{M}}^{*}\right)$ and apply (3.1) to the field $d v$

$$
d v=P I^{*} I d v+d S \delta d v+R d v .
$$

Since $I d v=0$, this gives $d S \delta d v=d v-R d v$. Applying the operator $\delta$ to the latter equality, we obtain $\delta d S \delta d=\delta d-\delta R d$. This implies that $S$ is a parametrix for $\delta d$.

The arguments of the previous paragraph show that $I^{*} I d=0$. So, the operator $I^{*} I$ is not elliptic and has no parametrix in the case of $m>0$. Nevertheless, $P I^{*} I f=f-R f$ for any solenoidal field $f$. Therefore we call the operator $P$ the parametrix of $I^{*} I$ on the space of solenoidal fields.

We will first reduce the theorem to the following weaker statement.

Lemma 3.2 There exist pseudodifferential operators $P_{1}$ and $B$ of orders 1 and -1 respectively such that

$$
E=P_{1} I^{*} I+d S \delta+B
$$

Proof of Theorem 3.1. Assume Lemma 3.2 to be valid. We multiply (3.2) by $d$ from the right and then use that $S$ is a parametrix of $\delta d$ and $I^{*} I d=0$. We obtain

$$
B d=R_{1}
$$

with a smoothing operator $R_{1}$.

$C=\sum_{k=0}^{\infty} B^{k}$ is a well defined pseudodifferential operator of order 0 and it is a parametrix for $E-B$. Rewrite (3.2) as

$$
P_{1} I^{*} I+d S \delta=E-B
$$

and compose on the left the latter equality with $C$

$$
C P_{1} I^{*} I+d S \delta+\sum_{k=1}^{\infty} B^{k} d S \delta=C(E-B)=E+R_{2}
$$

with a smoothing $R_{2}$. By (3.3), the last sum on the left-hand side is a smoothing operator. We have thus obtained (3.1) with $P=C P_{1}$.

Proof of Lemma 3.2. First of all we observe that the statement is of a pure algebraic nature. Let $p(\xi)=\sigma_{1}\left(P_{1}\right)$ be the principal symbol of the operator $P_{1}$ we are looking for. Equation (3.2) is equivalent to the following one:

$$
E=p(\xi) \lambda(\xi)+i_{\xi}\left(j_{\xi} i_{\xi}\right)^{-1} j_{\xi}
$$

So, we have to find an operator $p(\xi): S^{m}\left(T_{x}^{*} \tilde{M}\right) \rightarrow S^{m}\left(T_{x}^{*} \tilde{M}\right)$ such that

$$
f=p(\xi) \lambda(\xi) f+i_{\xi}\left(j_{\xi} i_{\xi}\right)^{-1} j_{\xi} f
$$

for any $f \in S^{m}\left(T_{x}^{*} \tilde{M}\right)$ and for any $0 \neq \xi \in T_{x}^{*} \tilde{M}$. The point $x \in \tilde{M}$ is fixed in the proof.

Since $i_{\xi}$ and $j_{\xi}$ are dual to each other, any tensor $f \in S^{m}\left(T_{x}^{*} \tilde{M}\right)$ can be uniquely represented in the form

$$
f=\tilde{f}+i_{\xi} v, \quad j_{\xi} \tilde{f}=0
$$


with $\tilde{f} \in S^{m}\left(T_{x}^{*} \tilde{M}\right)$ and $v \in S^{m-1}\left(T_{x}^{*} \tilde{M}\right)$. Substituting the expression into (3.4), we have

$$
\tilde{f}+i_{\xi} v=p(\xi) \lambda(\xi)\left(\tilde{f}+i_{\xi} v\right)+i_{\xi}\left(j_{\xi} i_{\xi}\right)^{-1} j_{\xi}\left(\tilde{f}+i_{\xi} v\right) .
$$

Since $\lambda(\xi) i_{\xi}=0$, the latter formula is equivalent to

$$
\tilde{f}=p(\xi) \lambda(\xi) \tilde{f}
$$

In other words, the operator $p(\xi) \lambda(\xi)$, restricted to the space

$$
S_{\xi}^{m}\left(T_{x}^{*} \tilde{M}\right)=\left\{f \in S^{m}\left(T_{x}^{*} \tilde{M}\right) \mid j_{\xi} f=0\right\}
$$

must be the identity.

By $(2.8), \lambda(\xi)$ maps $S^{m}\left(T_{x}^{*} \tilde{M}\right)$ to $S_{\xi}^{m}\left(T_{x}^{*} \tilde{M}\right)$. Therefore the existence of an operator $p(\xi)$ satisfying (3.4) is equivalent to the statement: the operator

$$
\lambda(\xi): S_{\xi}^{m}\left(T_{x}^{*} \tilde{M}\right) \rightarrow S_{\xi}^{m}\left(T_{x}^{*} \tilde{M}\right)
$$

is an isomorphism for any $\xi \neq 0$. The latter statement is proved in Theorem 2.12.1 of [7]. It does not matter that the case of Euclidean space is considered there because, for a fixed point $x \in \tilde{M}$, we can identify $T_{x}^{*} \tilde{M}$ with $\mathbf{R}^{n}$ with the help of an orthonormal basis. The lemma is proved.

In Theorem 2.12.1 of [7], the following explicit formula for $p(\xi)$ is obtained:

$$
p(\xi)=|\xi| \sum_{k=0}^{[m / 2]} c(k, m, n) i_{\varepsilon}^{k} j_{g}^{k},
$$

where $j_{g}$ is the contraction with the metric tensor $g, i_{\varepsilon}$ is the symmetric multiplication by the tensor $\varepsilon=\left(\varepsilon_{i j}(\xi)\right), n=\operatorname{dim} M$, and the coefficients are expressed by

$$
c(k, m, n)=(-1)^{k} \frac{\Gamma(n / 2-1 / 2)}{2 \sqrt{\pi}(n-3) ! ! \Gamma(n / 2)} \frac{(n+2 m-2 k-3) ! !}{2^{k} k !(m-2 k) !} .
$$

Observe that our proof of Theorem 3.1 gives an explicit procedure for constructing the parametrix $P$ as in (3.1). Indeed, we first construct the operator $P_{1}$ with the principal symbol $p(\xi)$ defined by (3.5)-(3.6). Then we define the operator $B$ by formula (3.2) and construct $C=\sum_{k=0}^{\infty} B^{k}$. Finally, we set $P=C P_{1}$.

Multiplying equation (3.1) by $\delta$ from the left, we obtain

$$
\delta P I^{*} I=R_{1}
$$

with a smoothing operator $R_{1}$. This means that the tensor field $P I^{*} I f$ is solenoidal, up to a smooth field, for any $f$. Let $\tilde{f}$ be the solenoidal part of $f \in L^{2}\left(S^{m} \tau_{M}^{*}\right)$. Later, in Section 5, we will show that the difference $P I^{*} I f-\tilde{f}$ is smooth on $M \backslash \partial M$. This gives the explicit formula for reconstructing the wave front set

$$
W F\left(\left.\tilde{f}\right|_{M \backslash \partial M}\right)=W F\left(\left.P I^{*} I f\right|_{M \backslash \partial M}\right) .
$$




\section{Tensor field-distributions and the transmission condition}

Let $(M, g)$ be an open Riemannian manifold. By $\mathcal{D}\left(S^{m} \tau_{M}^{*}\right)$ we denote the space $C_{0}^{\infty}\left(S^{m} \tau_{M}^{*}\right)$ endowed with the corresponding topology, and by $\mathcal{D}^{\prime}\left(S^{m} \tau_{M}^{*}\right)$ we denote the dual space. Elements of $\mathcal{D}\left(S^{m} \tau_{M}^{*}\right)$ are called test tensor fields while elements of $\mathcal{D}^{\prime}\left(S^{m} \tau_{M}^{*}\right)$ are called (symmetric covariant) tensor field-distributions of rank $m$. The value of a functional $f \in \mathcal{D}^{\prime}\left(S^{m} \tau_{M}^{*}\right)$ on a test field $u \in \mathcal{D}\left(S^{m} \tau_{M}^{*}\right)$ is denoted by $\langle f \mid u\rangle$. The notations $\mathcal{D}\left(S^{0} \tau_{M}^{*}\right)$ and $\mathcal{D}^{\prime}\left(S^{0} \tau_{M}^{*}\right)$ will be abbreviated to $\mathcal{D}(M)$ and $\mathcal{D}^{\prime}(M)$ respectively. The embedding $C^{\infty}\left(S^{m} \tau_{M}^{*}\right) \subset \mathcal{D}^{\prime}\left(S^{m} \tau_{M}^{*}\right)$ is defined by

$$
\langle f \mid u\rangle=\int_{M}\langle f, \bar{u}\rangle d V^{n} \quad\left(u \in \mathcal{D}\left(S^{m} \tau_{M}^{*}\right)\right),
$$

where $d V^{n}$ is the Riemannian volume form. For $f \in \mathcal{D}^{\prime}\left(S^{m} \tau_{M}^{*}\right)$ and $u \in C^{\infty}\left(S^{m} \tau_{M}^{*}\right)$, the dot product $\langle f, u\rangle \in \mathcal{D}^{\prime}(M)$ is well defined.

If $\left(x^{1}, \ldots, x^{n}\right)$ is a local coordinate system with the domain $U \subset M$, then the coordinates $f_{i_{1} \ldots i_{m}} \in \mathcal{D}^{\prime}(U)$ are defined for $f \in \mathcal{D}^{\prime}\left(S^{m} \tau_{M}^{*}\right)$. Formula (4.1) can be written in coordinates as follows:

$$
\langle f \mid u\rangle=\int_{U} f_{i_{1} \ldots i_{m}} u^{i_{1} \ldots i_{m}} \sqrt{g} d x^{1} \ldots d x^{n} \quad\left(u \in \mathcal{D}\left(S^{m} \tau_{M}^{*}\right), \operatorname{supp} u \in U\right),
$$

where $g=\operatorname{det}\left(g_{i j}\right)$.

Let $\Gamma$ be a smooth hypersurface in $M$ which is considered as an $(n-1)$-dimensional Riemannian manifold with the metric induced from $M$, and $\left.S^{m} \tau_{M}^{*}\right|_{\Gamma}$ be the restriction of the bundle $S^{m} \tau_{M}^{*}$ to $\Gamma$. Define the operator

$$
\mathcal{D}^{\prime}\left(\left.S^{m} \tau_{M}^{*}\right|_{\Gamma}\right) \rightarrow \mathcal{D}^{\prime}\left(S^{m} \tau_{M}^{*}\right), \quad f \mapsto f \otimes \delta_{\Gamma}
$$

by the formula

$$
\left\langle f \otimes \delta_{\Gamma} \mid u\right\rangle=\int_{\Gamma}\langle f, \bar{u}\rangle d V^{n-1} \quad\left(u \in \mathcal{D}\left(S^{m} \tau_{M}^{*}\right)\right),
$$

where $d V^{n-1}$ is the Riemannian volume form of $\Gamma$. In semigeodesic coordinates (such that $\left|x^{n}\right|$ coincides with the distance from $x$ to $\Gamma$ ) this operator looks as follows:

$$
\left(f \otimes \delta_{\Gamma}\right)_{i_{1} \ldots i_{m}}=f_{i_{1} \ldots i_{m}}\left(x^{1}, \ldots, x^{n-1}\right) \delta_{0}\left(x^{n}\right),
$$

where $\delta_{0}$ stands for the Dirac delta function.

We will use the transmission condition in the following weak form, compare with Section 2 of Chapter 5 of [2]. A classical pseudodifferential operator $A$ of order $m$ is said to satisfy the transmission condition if, in the asymptotic expansion $\sigma(A) \sim \sum_{j=m}^{-\infty} a_{j}(x, \xi)$ of the full symbol, each term $a_{j}(x, \xi)$ is a rational function of $\xi$ which is homogeneous of degree $j$.

The following two lemmas coincide, up to nonrelevant details, with Theorems 2.2 and 2.4 of Chapter 5 of $[2]$. 
Lemma 4.1 Let $\Omega \subset M$ be an open regular domain bounded by a smooth hypersurface $\Gamma=\partial \Omega$, and let $A: C_{0}^{\infty}\left(S^{m} \tau_{M}^{*}\right) \rightarrow C^{\infty}\left(S^{m} \tau_{M}^{*}\right)$ be a classical pseudodifferential operator on the bundle $S^{m} \tau_{M}^{*}$ satisfying the transmission condition. If a compactly supported fielddistribution $u \in \mathcal{D}^{\prime}\left(S^{m} \tau_{M}^{*}\right)$ satisfies $\left.u\right|_{\Omega}=0$, then $\left.(A u)\right|_{\Omega}$ has restrictions of all orders to $\Gamma$.

The latter statement should be supplemented with the following definition. In the setting of the lemma, a distribution $v \in \mathcal{D}^{\prime}(\Omega)$ has restrictions of order $k$ to $\Gamma$ if the restriction $\left.v\right|_{U}$ to the domain $U \subset \Omega$ of any semigeodesic coordinate system $\left(x^{1}, \ldots, x^{n}\right)$ with $x^{n}=\operatorname{dist}(x, \Gamma)$ is a $C^{k}$-function of $x^{n} \geq 0$ with values in the space of distributions of variables $\left(x^{1}, \ldots, x^{n-1}\right)$.

Lemma 4.2 (Boundary regularity of a surface potential). Let $\Omega \subset M$ be an open regular domain bounded by a smooth hypersurface $\Gamma=\partial \Omega$, and let $A: C_{0}^{\infty}\left(S^{m} \tau_{M}^{*}\right) \rightarrow C^{\infty}\left(S^{m} \tau_{M}^{*}\right)$ be a classical pseudodifferential operator of order $\mu$ satisfying the transmission condition. Then

1. The operator $K$ defined by $K f=\left.\left[A\left(f \otimes \delta_{\Gamma}\right)\right]\right|_{\Omega}$ is continuous from $C_{0}^{\infty}\left(\left.S^{m} \tau_{M}^{*}\right|_{\Gamma}\right)$ to $C^{\infty}\left(\left.S^{m} \tau_{M}^{*}\right|_{\bar{\Omega}}\right)$.

2. The operator

$$
C: C_{0}^{\infty}\left(\left.S^{m} \tau_{M}^{*}\right|_{\Gamma}\right) \rightarrow C^{\infty}\left(\left.S^{m} \tau_{M}^{*}\right|_{\Gamma}\right), \quad C f=\left.(K f)\right|_{\Gamma}
$$

is the pseudodifferential operator of order $\mu+1$ on $\Gamma$.

\section{$5 \quad$ Regularity of nontrivial ghosts}

In this section $M$ is again a simple compact Riemannian manifold with boundary embedded into a simple open Riemannian manifold $\tilde{M}$. The interior $\stackrel{\circ}{M}=M \backslash \partial M$ of $M$ is the open regular domain in $\tilde{M}$ bounded by the smooth compact hypersurface $\partial \stackrel{\circ}{M}=\partial M \subset \tilde{M}$.

First of all we prove smoothness of a ghost in $\stackrel{\circ}{M}$.

Theorem 5.1 Let $f \in L^{2}\left(S^{m} \tau_{M}^{*}\right)$ satisfy If $=0$ and $\delta f=0$. Then $f$ is $C^{\infty}$-smooth in $\stackrel{\circ}{M}$ and $\left.f\right|_{M}$ has restrictions of all orders to $\partial M$.

Proof. Extend $f$ by zero to $\tilde{M} \backslash M$ and denote the extension by $f^{\circ}$. Then at least $f^{\circ} \in L^{2}\left(S^{m} \tau_{\tilde{M}}^{*}\right)$. The extension is still in the kernel of the ray transform, $I f^{\circ}=0$, where $I$ is now the ray transform on $\tilde{M}$. Apply Theorem 3.1 to $f^{\circ}$

$$
f^{\circ}=d S \delta f^{\circ}+R f^{\circ}
$$

$\delta f^{\circ}$ is the tensor field-distribution on $\tilde{M}$ supported on $\partial M$. Therefore the restriction of the first term on the right-hand side of $(5.1)$ to $\stackrel{\circ}{M}$ is smooth. The second term is smooth on the whole of $\tilde{M}$. Now (5.1) implies smoothness of $\left.f^{\circ}\right|_{M} ^{\circ}=\left.f\right|_{M}$. This proves the first statement of the theorem.

The operator $d S$ satisfies the transmission condition. By the hypothesis of the theorem, $\left.\left(\delta f^{\circ}\right)\right|_{M} ^{\circ}=\left.(\delta f)\right|_{M} ^{\circ}=0$. Applying Lemma 4.1 , we see that $\left.\left(d S \delta f^{\circ}\right)\right|_{M} ^{\circ}$ has restrictions of any order to $\partial M$. By (5.1), the same is true for $\left.f\right|_{M}$. The theorem is proved. 
To finish the proof of Theorem 1.1, it remains to prove smoothness of the trace $\left.\operatorname{tr}\right|_{\partial M} f=\left.f\right|_{\partial M}$. To this end we first calculate $\delta f^{\circ}$.

Lemma 5.2 Let $f \in L^{2}\left(S^{m} \tau_{M}^{*}\right)$ satisfy $I f=0$ and $\delta f=0$, and let $f^{\circ}$ be the extension of $f$ to $\tilde{M}$ as zero outside $M$. Then

$$
\delta f^{\circ}=\left.j_{\nu} f\right|_{\partial M} \otimes \delta_{\partial M},
$$

where $\nu$ is the unite inner normal to the boundary.

Proof. By Theorem 5.1, the trace $\left.f\right|_{\partial M}$ is a well defined tensor field-distribution on $\partial M$. For a test field $u \in \mathcal{D}\left(S^{m-1} \tau_{\tilde{M}}^{*}\right)$,

$$
\left\langle\delta f^{\circ} \mid u\right\rangle=-\left\langle f^{\circ} \mid d u\right\rangle=-\int_{M}\langle f, d \bar{u}\rangle d V^{n}
$$

By Green's formula (see Theorem 3.3.1 of [7]),

$$
\int_{M}(\langle f, d \bar{u}\rangle+\langle\delta f, \bar{u}\rangle) d V^{n}=-\int_{\partial M}\left\langle j_{\nu} f, \bar{u}\right\rangle d V^{n-1} .
$$

Since $\delta f=0$, then the last two equations give

$$
\left\langle\delta f^{\circ} \mid u\right\rangle=\int_{\partial M}\left\langle j_{\nu} f, \bar{u}\right\rangle d V^{n-1}=\left\langle\left. j_{\nu} f\right|_{\partial M} \otimes \delta_{\partial M} \mid u\right\rangle .
$$

The lemma is proved.

Remark. The hypothesis If $=0$ of Lemma 5.2 can be replaced with $f \in H^{1}\left(S^{m} \tau_{M}^{*}\right)$. The trace $\left.f\right|_{\partial M} \in L^{2}\left(\left.S^{m} \tau_{M}^{*}\right|_{\partial M}\right)$ is well defined under the latter hypothesis, and the rest of the proof is the same.

Proof of Theorem 1.1. Let $f \in L^{2}\left(S^{m} \tau_{M}^{*}\right)$ satisfy $\delta f=0$ and $I f=0$, and $f^{\circ}$ be the extension of $f$ to $\tilde{M}$, as zero outside $M$. Substituting the value (5.2) for $\delta f^{\circ}$ into (5.1), we obtain

$$
f^{\circ}=d S\left(\left.j_{\nu} f\right|_{\partial M} \otimes \delta_{\partial M}\right)+R f^{\circ} .
$$

By $\left.\operatorname{tr}\right|_{\partial M}$ we mean below the limit value from the inner part of $M$. We apply the operator $\left.j_{\nu} \operatorname{tr}\right|_{\partial M}$ to $(5.3)$

$$
\left.j_{\nu} f\right|_{\partial M}=\left.j_{\nu} \operatorname{tr}\right|_{\partial M} d S\left(\left.j_{\nu} f\right|_{\partial M} \otimes \delta_{\partial M}\right)+\left.j_{\nu} \operatorname{tr}\right|_{\partial M} R f^{\circ} .
$$

We rewrite this equality in the form

$$
\left.j_{\nu} f\right|_{\partial M}=\mathcal{A}\left(\left.j_{\nu} f\right|_{\partial M}\right)+\left.j_{\nu} \operatorname{tr}\right|_{\partial M} R f^{\circ},
$$

where the operator

$$
\mathcal{A}: C^{\infty}\left(\left.S^{m-1} \tau_{M}^{*}\right|_{\partial M}\right) \rightarrow C^{\infty}\left(\left.S^{m-1} \tau_{M}^{*}\right|_{\partial M}\right)
$$

is defined by

$$
\mathcal{A} u=\left.j_{\nu} \operatorname{tr}\right|_{\partial M} d S\left(u \otimes \delta_{\partial M}\right) .
$$


By Lemma $4.2, \mathcal{A}$ is a pseudodifferential operator of order 0 on $\partial M$. We have thus proved that the field $h=\left.j_{\nu} f\right|_{\partial M}$ satisfies the equation

$$
(E-\mathcal{A}) h=\left.j_{\nu} \operatorname{tr}\right|_{\partial M} R f^{\circ}
$$

with a smooth right-hand side, where $E$ is the identity operator. The ellipticity of $E-\mathcal{A}$ would imply the smoothness of $h=\left.j_{\nu} f\right|_{\partial M}$. If this is the case, using equation (5.3) and Lemma 4.2, we would then prove smoothness of the field $\left.f^{\circ}\right|_{\partial M}=\left.f\right|_{\partial M}$.

Thus, Theorem 1.1 is reduced to the following

Lemma 5.3 The operator

$$
E-\mathcal{A}: C^{\infty}\left(\left.S^{m} \tau_{M}^{*}\right|_{\partial M}\right) \rightarrow C^{\infty}\left(\left.S^{m} \tau_{M}^{*}\right|_{\partial M}\right)
$$

is elliptic for any integer $m \geq 0$. Here $E$ is the identity and $\mathcal{A}$ is defined by (5.4).

The following statement is the basis for the wave front set reconstruction procedure that has been discussed in Section 3 .

Theorem 5.4 Let $P$ be the operator constructed in Theorem 3.1. For $f \in L^{2}\left(S^{m} \tau_{M}^{*}\right)$, if

$$
f=\tilde{f}+d v, \quad \delta \tilde{f}=0,\left.\quad v\right|_{\partial M}=0
$$

is the decomposition into solenoidal and potential parts, then the field $P I^{*} I f-\tilde{f}$ is smooth in $\stackrel{\circ}{M}$.

Proof. Extend $f, \tilde{f}, v$ by zero to $\tilde{M} \backslash M$ and denote the extensions by $f^{\circ}, \tilde{f}^{\circ}, v^{\circ}$ respectively. Then If $f^{\circ}=I f$ and the difference $h=f^{\circ}-\tilde{f}^{\circ}-d v^{\circ}$ is a tensor fielddistribution supported in $\partial M$. Apply Theorem 3.1 to $f^{\circ}$

$$
f^{\circ}=P I^{*} I f+d S \delta f^{\circ}+R f^{\circ} .
$$

Substitute the expression $f^{\circ}=\tilde{f}^{\circ}+d v^{\circ}+h$ into this equation

$$
\tilde{f}^{\circ}+d v^{\circ}+h=P I^{*} I f+d S \delta\left(\tilde{f}^{\circ}+d v^{\circ}+h\right)+R f^{\circ} .
$$

Since $d S \delta d v^{\circ}$ coincides with $d v^{\circ}$ up to a smooth field, the latter equation is simplified to the following one:

$$
\tilde{f}^{\circ}-P I^{*} I f=d S \delta \tilde{f}^{\circ}-h+d S \delta h+R_{1} f^{\circ},
$$

where $R_{1}$ is a smoothing operator. Take the restriction of the latter equation to $\stackrel{\circ}{M}$. Restrictions of all terms on the right-hand side are smooth. So we have obtained that $\left.\left(\tilde{f}^{\circ}-P I^{*} I f\right)\right|_{M} ^{\circ}=\left.\left(\tilde{f}-P I^{*} I f\right)\right|_{M} ^{\circ}$ is a smooth field. The theorem is proved.

The rest of the article is devoted to the proof of Lemma 5.3. 


\section{Symbol of the operator $\mathcal{A}$}

Here we calculate the principal symbol $A=\sigma_{0}(\mathcal{A})$ of the operator $\mathcal{A}$. Choose a semigeodesic coordinate system $\left(x^{1}, \ldots, x^{n}\right)$ in a neighborhood $U \subset \tilde{M}$ of a point $x_{0} \in \partial M$ such that $\left|x^{n}\right|=\operatorname{dist}(x, \partial M)$ and $x^{n}>0$ in $U \cap \stackrel{\circ}{M}$. In such coordinates

$$
\mathcal{A} f=j_{\nu} \lim _{x^{n} \rightarrow+0} d S\left(f \otimes \delta_{0}\left(x^{n}\right)\right),
$$

where $\nu$ is the inner unit normal to $\partial M$ and $\delta_{0}$ is the Dirac delta function.

Let $b(x, \xi)=\sigma(d S)$ be the full symbol of $d S$ in the chosen coordinates, and $b_{-1}=$ $\sigma_{-1}(d S)$ be its principal symbol. Since $S$ is a parametrix of $\delta d$,

$$
\sigma_{-2}(S)=-\left(j_{\xi} i_{\xi}\right)^{-1}=-\sum_{k=0}^{m}(-1)^{k}\left(\begin{array}{c}
m+1 \\
k+1
\end{array}\right) \frac{i_{\xi}^{k} j_{\xi}^{k}}{|\xi|^{2 k+2}}
$$

where $\left(\begin{array}{c}m+1 \\ k+1\end{array}\right)$ is the binomial coefficient. The last equality is written using Lemma 7.6 that will be proved later. From the latter formula,

$$
b_{-1}=-i \sum_{k=0}^{m}(-1)^{k}\left(\begin{array}{c}
m+1 \\
k+1
\end{array}\right) \frac{i_{\xi}^{k+1} j_{\xi}^{k}}{|\xi|^{2 k+2}} .
$$

Let $f \in C_{0}^{\infty}\left(\left.S^{m} \tau_{M}^{*}\right|_{\partial M}\right)$ be supported in $U \cap \partial M$. By the definition of a pseudodifferential operator

$$
d S\left(f \otimes \delta_{0}\right)(x)=(2 \pi)^{-n} \int e^{i\langle x, \xi\rangle} b(x, \xi) f \widehat{\otimes} \delta_{0}(\xi) d \xi .
$$

In what follows we use the notation $\xi=\left(\xi^{\prime}, \xi_{n}\right)$ with $\xi^{\prime}=\left(\xi_{1}, \ldots, \xi_{n-1}\right)$. Since $f \widehat{\otimes} \delta_{0}(\xi)=$ $\widehat{f}\left(\xi^{\prime}\right)$, the previous formula can be rewritten as follows:

$$
d S\left(f \otimes \delta_{0}\right)(x)=(2 \pi)^{1-n} \int e^{i\left\langle x^{\prime}, \xi^{\prime}\right\rangle}\left(\frac{1}{2 \pi} \int_{-\infty}^{\infty} e^{i x^{n} \xi_{n}} b(x, \xi) d \xi_{n}\right) \widehat{f}\left(\xi^{\prime}\right) d \xi^{\prime}
$$

This means that the symbol $\tilde{b}$ of the operator $\left.f \mapsto\left[d S\left(f \otimes \delta_{0}\left(x^{n}\right)\right)\right]\right|_{\partial M}$ is given by

$$
\tilde{b}\left(x, \xi^{\prime}\right)=\lim _{x^{n} \rightarrow+0} \frac{1}{2 \pi} \int_{-\infty}^{\infty} e^{i x^{n} \xi_{n}} b(x, \xi) d \xi_{n} .
$$

Let $A$ be the principal symbol of $\mathcal{A}$. Formulas (6.1)-(6.3) imply that

$$
A\left(\xi^{\prime}\right)=j_{\nu} \sum_{k=0}^{m}(-1)^{k}\left(\begin{array}{c}
m+1 \\
k+1
\end{array}\right) \lim _{x^{n} \rightarrow+0} \frac{1}{2 \pi i} \int_{-\infty}^{\infty} e^{i x^{n} \xi_{n}} \frac{i_{\xi}^{k+1} j_{\xi}^{k}}{|\xi|^{2 k+2}} d \xi_{n} .
$$

We are going to evaluate the integral in (6.4) by the method of residues. To this end we consider $f\left(\xi_{n}\right)=i_{\xi}^{k+1} j_{\xi}^{k} /|\xi|^{2 k+2}$ as a function of the complex argument $\xi_{n}$. It is the meromorphic function in the half-plane $\operatorname{Im} \xi_{n}>0$ with the unique pole at $\xi_{n}=i\left|\xi^{\prime}\right|$ of order $k+1$. By the Jordan lemma,

$$
\frac{1}{2 \pi i} \int_{-\infty}^{\infty} e^{i x^{n} \xi_{n}} \frac{i_{\xi}^{k+1} j_{\xi}^{k}}{|\xi|^{2 k+2}} d \xi_{n}=\left.\operatorname{Res}\left(e^{i x^{n} \xi_{n}} \frac{i_{\xi}^{k+1} j_{\xi}^{k}}{|\xi|^{2 k+2}}\right)\right|_{\xi_{n}=i\left|\xi^{\prime}\right|}
$$


for $x^{n}>0$. We use the following rule for evaluating residues: if $f(z)=\varphi(z) /(\psi(z))^{k+1}$, where $\varphi(z)$ and $\psi(z)$ are holomorphic functions in a neighborhood of $z_{0}$ with $\psi\left(z_{0}\right)=0$ and $\psi^{\prime}\left(z_{0}\right) \neq 0$, then

$$
\operatorname{Res}\left[\frac{\varphi(z)}{(\psi(z))^{k+1}}\right]_{z=z_{0}}=\frac{1}{k !} \frac{\varphi^{(k)}\left(z_{0}\right)}{\left(\psi^{\prime}\left(z_{0}\right)\right)^{k+1}} .
$$

Applying this rule, we obtain from (6.5)

$$
\frac{1}{2 \pi i} \int_{-\infty}^{\infty} e^{i x^{n} \xi_{n}} \frac{i_{\xi}^{k+1} j_{\xi}^{k}}{|\xi|^{2 k+2}} d \xi_{n}=\left.\frac{1}{k !\left(2 i\left|\xi^{\prime}\right|\right)^{k+1}} \frac{d^{k}}{d \xi_{n}^{k}}\left(e^{i x^{n} \xi_{n}} i_{\xi}^{k+1} j_{\xi}^{k}\right)\right|_{\xi_{n}=i\left|\xi^{\prime}\right|}
$$

This implies

$$
\lim _{x^{n} \rightarrow+0} \frac{1}{2 \pi i} \int_{-\infty}^{\infty} e^{i x^{n} \xi_{n}} \frac{i_{\xi}^{k+1} j_{\xi}^{k}}{|\xi|^{2 k+2}} d \xi_{n}=\left.\frac{1}{k !\left(2 i\left|\xi^{\prime}\right|\right)^{k+1}} \frac{d^{k}}{d \xi_{n}^{k}}\left(i_{\xi}^{k+1} j_{\xi}^{k}\right)\right|_{\xi_{n}=i\left|\xi^{\prime}\right|} .
$$

Substituting this value into (6.4), we obtain the final formula for the principal symbol $A$ of the operator $\mathcal{A}$

$$
A\left(\xi^{\prime}\right)=\left.j_{\nu} \sum_{k=0}^{m} \frac{(-1)^{k}}{k !}\left(\begin{array}{c}
m+1 \\
k+1
\end{array}\right) \frac{1}{\left(2 i\left|\xi^{\prime}\right|\right)^{k+1}} \frac{d^{k}}{d \xi_{n}^{k}}\left(i_{\xi}^{k+1} j_{\xi}^{k}\right)\right|_{\xi_{n}=i\left|\xi^{\prime}\right|} .
$$

Formula (6.6) has a pure algebraic character. Indeed, since $i_{\xi}=i_{\xi^{\prime}}+\xi_{n} i_{\nu}$ and $j_{\xi}=$ $j_{\xi^{\prime}}+\xi_{n} j_{\nu}$, the product $i_{\xi}^{k+1} j_{\xi}^{k}$ is a polynomial of degree $2 k+1$ in $\xi_{n}$ with operator-valued coefficients.

\section{Decomposition of a tensor}

The rest of the paper is of a pure algebraic nature. We will analyze the operator (6.6) at a point $x \in \partial M$ which is fixed from now on so that we will omit all the dependence on $x$. Our goal is to prove that

$$
E-A\left(\xi^{\prime}\right): S^{m}\left(T_{x}^{*} M\right) \rightarrow S^{m}\left(T_{x}^{*} M\right)
$$

is an isomorphism for any covector $0 \neq \xi^{\prime} \in T_{x}^{*} M$ which is orthogonal to the vector $\nu$. We denote $V=T_{x} M$ and identify $V$ with its dual $V^{*}$ using the Riemannian scalar product. Thus, $V$ is an $n$-dimensional real vector space endowed with a scalar product $\langle\cdot, \cdot\rangle$ and the corresponding norm $|\cdot|$. A unit vector $\nu \in V$ is distinguished which is the inner normal to the boundary. Let $S^{m}=S^{m}(V)$ be the space of complex symmetric $m$-tensors on $V$.

In this section we will establish some commutator formulas for the operators involved in (6.6), and will return to analyzing operator (6.6) in the next section.

Lemma 7.1 Given two vectors $u, v \in V$, the formula

$$
j_{u}^{k} i_{v}^{l}=\sum_{p=\max (0, k-l)}^{\min (k, m)} \frac{\left(\begin{array}{c}
m \\
p
\end{array}\right)\left(\begin{array}{c}
l \\
k-p
\end{array}\right)}{\left(\begin{array}{c}
m+l \\
k
\end{array}\right)}\langle u, v\rangle^{k-p} i_{v}^{p+l-k} j_{v}^{p}
$$

is valid on $S^{m}$ for $k \leq m+l$. 
For $k=1$ we obtain

Corollary 7.2 For two vectors $u, v \in V$, the formula

$$
j_{u} i_{v}^{l}=\frac{l}{m+l}\langle u, v\rangle i_{v}^{l-1}+\frac{m}{m+l} i_{v}^{l} j_{u}
$$

is valid on $S^{m}$ if $m+l>0$.

Corollary 7.3 If $\langle u, v\rangle=0$, then the formula

$$
j_{u}^{k} i_{v}^{l}=\left\{\begin{array}{l}
0 \quad \text { for } \quad k>m, \\
\frac{\left(\begin{array}{c}
m \\
k
\end{array}\right)}{\left(\begin{array}{c}
m+l \\
k
\end{array}\right)} i_{v}^{l} j_{u}^{k} \quad \text { for } \quad k \leq m
\end{array}\right.
$$

holds on $S^{m}$ if $k \leq m+l$.

Corollary 7.4 If $j_{u} f=0$ for $f \in S^{m}$, then

$$
j_{u}^{k} i_{v}^{l} f=\left\{\begin{array}{l}
0 \quad \text { for } \quad k>l, \\
\frac{\left(\begin{array}{l}
l \\
k
\end{array}\right)}{\left(\begin{array}{c}
m+l \\
k
\end{array}\right)}\langle u, v\rangle^{k} i_{v}^{l-k} f \quad \text { for } \quad k \leq l .
\end{array}\right.
$$

Sketch of the proof of Lemma 7.1. In the case of $k=l=1$ formula (7.2) looks as follows:

$$
j_{u} i_{v}=\frac{\langle u, v\rangle}{m+1} E+\frac{m}{m+1} i_{v} j_{u}
$$

where $E$ is the identity operator. The latter formula is proved by a straightforward calculation in coordinates. For $u=v$, this calculation is presented at the end of Section 3.3 of [7]. Starting with (7.3), one easily proves (7.2) for $k=1$ by induction on $l$, and then proves (7.2) by induction on $k$ in the general case.

Lemma 7.5 Let $u$ and $v$ be two nonzero vectors in $V$ which are orthogonal to each other, $\langle u, v\rangle=0$. Any tensor $f \in S^{m}$ can be uniquely represented in the form

$$
f=\sum_{r+s \leq m} i_{u}^{r} i_{v}^{s} f_{r s}
$$

where $f_{r s} \in S^{m-r-s}$ satisfies

$$
j_{u} f_{r s}=j_{v} f_{r s}=0 .
$$

The summands of (7.4) are orthogonal to each other. In other words, we have the orthogonal decomposition

$$
S^{m}=\bigoplus_{r+s \leq m} S_{r s}^{m}(u, v),
$$

where $S_{r s}^{m}(u, v)$ is the subspace of $S^{m}$ consisting of tensors $f$ that can be represented in the form $f=i_{u}^{r} i_{v}^{s} g$ with some $g \in S^{m-r-s}$ satisfying $j_{u} g=j_{v} g=0$. 
Proof. We first prove uniqueness of the representation (7.4). Fix some $r_{0}, s_{0}$ and apply the operator $j_{u}^{r_{0}} j_{v}^{s_{0}}$ to $(7.4)$

$$
j_{u}^{r_{0}} j_{v}^{s_{0}} f=\sum_{r+s \leq m} j_{u}^{r_{0}} j_{v}^{s_{0}} i_{u}^{r} i_{v}^{s} f_{r s}
$$

Let us recall that the operators $i_{u}$ and $i_{v}$ commute for any $u$ and $v$, as well as $j_{u}$ and $j_{v}$ commute.

In the expression $j_{u}^{r_{0}} j_{v}^{s_{0}} i_{u}^{r} i_{v}^{s} f_{r s}$ from (7.6), we first transpose the operators $j_{v}^{s_{0}}$ and $i_{u}^{r}$. Since $\langle u, v\rangle=0$, Corollary 7.3 gives

$$
j_{u}^{r_{0}}\left(j_{v}^{s_{0}} i_{u}^{r}\right) i_{v}^{s} f_{r s}=\frac{\left(\begin{array}{c}
m-s_{0} \\
r
\end{array}\right)}{\left(\begin{array}{c}
m \\
r
\end{array}\right)} j_{u}^{r_{0}} i_{u}^{r} j_{v}^{s_{0}} i_{v}^{s} f_{r s} .
$$

Now, we transpose the factors $j_{v}^{s_{0}}$ and $i_{v}^{s}$ in the expression $j_{v}^{s_{0}} i_{v}^{s} f_{r s}$ using Lemma 7.1

$$
j_{v}^{s_{0}} i_{v}^{s} f_{r s}=\sum_{p=\max \left(0, s_{0}-s\right)}^{\min \left(s_{0}, m-r-s\right)} \frac{\left(\begin{array}{c}
s_{0} \\
p
\end{array}\right)\left(\begin{array}{c}
m-r-s_{0} \\
m-r-s-p
\end{array}\right)}{\left(\begin{array}{c}
m-r \\
s
\end{array}\right)}|v|^{2\left(s_{0}-p\right)} i_{v}^{p+s-s_{0}} j_{v}^{p} f_{r s} .
$$

If $s_{0}>s$, then the summation is over the positive values of $p$, and all summands on the right-hand side are equal to zero by (7.5). If $s_{0} \leq s$, then the right-hand side contains one nonzero summand corresponding to $p=0$. We have thus obtained

$$
j_{v}^{s_{0}} i_{v}^{s} f_{r s}=\left\{\begin{array}{l}
0 \quad \text { if } \quad s_{0}>s \\
\frac{\left(\begin{array}{c}
m-r-s_{0} \\
m-r-s
\end{array}\right)}{\left(\begin{array}{c}
m-r \\
s
\end{array}\right)}|v|^{2 s_{0}} i_{v}^{s-s_{0}} f_{r s} \quad \text { if } \quad s_{0} \leq s .
\end{array}\right.
$$

We substitute the latter expression into (7.7) to obtain

$$
j_{u}^{r_{0}} j_{v}^{s_{0}} i_{u}^{r} i_{v}^{s} f_{r s}=\left\{\begin{array}{l}
0 \quad \text { if } \quad s_{0}>s, \\
\frac{\left(\begin{array}{c}
m-s_{0} \\
r
\end{array}\right)\left(\begin{array}{c}
m-r-s_{0} \\
s-s_{0}
\end{array}\right)}{\left(\begin{array}{c}
m \\
r
\end{array}\right)\left(\begin{array}{c}
m-r \\
s
\end{array}\right)}|v|^{2 s_{0}} j_{u}^{r_{0}} i_{u}^{r} i_{v}^{s-s_{0}} f_{r s} \quad \text { if } \quad s_{0} \leq s .
\end{array}\right.
$$

Now, we transpose the factors $j_{u}^{r_{0}}$ and $i_{u}^{r}$ in the expression $j_{u}^{r_{0}} i_{u}^{r} i_{v}^{s-s_{0}} f_{r s}$. By Lemma 7.1 ,

$$
\left(j_{u}^{r_{0}} i_{u}^{r}\right) i_{v}^{s-s_{0}} f_{r s}=\sum_{p=\max \left(0, r_{0}-r\right)}^{\min \left(r_{0}, m-r-s_{0}\right)} \frac{\left(\begin{array}{c}
m-r-s_{0} \\
p
\end{array}\right)\left(\begin{array}{c}
r \\
r_{0}-p
\end{array}\right)}{\left(\begin{array}{c}
m-s_{0} \\
r_{0}
\end{array}\right)} i_{u}^{p+r-r_{0}} j_{u}^{p} i_{v}^{s-s_{0}} f_{r s} .
$$

By Corollary 7.3, the operators $j_{u}^{p}$ and $i_{v}^{s-s_{0}}$ commute up to a scalar factor. Together with (7.5), this implies that $j_{u}^{p} i_{v}^{s-s_{0}} f_{r s}=0$ for $p>0$. Therefore there can be only one nonzero summand corresponding to $p=0$ in the latter sum. We have thus obtained

$$
j_{u}^{r_{0}} i_{u}^{r} i_{v}^{s-s_{0}} f_{r s}=\left\{\begin{array}{l}
0 \quad \text { if } \quad r_{0}>r \\
\frac{\left(\begin{array}{c}
r \\
r_{0}
\end{array}\right)}{\left(\begin{array}{c}
m-s_{0} \\
r_{0}
\end{array}\right)}|u|^{2 r_{0}} i_{u}^{r-r_{0}} i_{v}^{s-s_{0}} f_{r s} \quad \text { if } \quad r_{0} \leq r .
\end{array}\right.
$$

We substitute the latter value into (7.8) to obtain, after the cancellation of factorials in the binomial coefficients,

$$
j_{u}^{r_{0}} j_{v}^{s_{0}} i_{u}^{r} i_{v}^{s} f_{r s}=\left\{\begin{array}{l}
0 \quad \text { if } r_{0}>r \quad \text { or } \quad s_{0}>s, \\
\frac{r_{0} ! s_{0} !\left(m-r_{0}-s_{0}\right) !}{m !}\left(\begin{array}{c}
r \\
r_{0}
\end{array}\right)\left(\begin{array}{c}
s \\
s_{0}
\end{array}\right)|u|^{2 r_{0}}|v|^{2 s_{0}} i_{u}^{r-r_{0}} i_{v}^{s-s_{0}} f_{r s} \quad \text { if } \quad r_{0} \leq r, s_{0} \leq s .
\end{array}\right.
$$


We substitute the latter expression into (7.6)

$$
j_{u}^{r_{0}} j_{v}^{s_{0}} f=|u|^{2 r_{0}}|v|^{2 s_{0}} \frac{r_{0} ! s_{0} !\left(m-r_{0}-s_{0}\right) !}{m !} \sum_{\substack{r+s \leq m \\
r \geq r_{0}, s \geq s_{0}}}\left(\begin{array}{c}
r \\
r_{0}
\end{array}\right)\left(\begin{array}{c}
s \\
s_{0}
\end{array}\right) i_{u}^{r-r_{0}} i_{v}^{s-s_{0}} f_{r s} .
$$

In the latter sum, we distinguish the summand corresponding to $(r, s)=\left(r_{0}, s_{0}\right)$ and obtain the recurrent formula

$$
f_{r_{0} s_{0}}=\frac{m !}{r_{0} ! s_{0} !\left(m-r_{0}-s_{0}\right) !|u|^{2 r_{0}}|v|^{2 s_{0}}} j_{u}^{r_{0}} j_{v}^{s_{0}} f-\sum_{\substack{r_{0}+s_{0}<r+s \leq m \\
r \geq r_{0}, s \geq s_{0}}}\left(\begin{array}{c}
r \\
r_{0}
\end{array}\right)\left(\begin{array}{c}
s \\
s_{0}
\end{array}\right) i_{u}^{r-r_{0}} i_{v}^{s-s_{0}} f_{r s}
$$

which determines uniquely all tensors $f_{r s}$.

Let us show that the summands of the decomposition (7.4) are orthogonal to each other. Let $(r, s) \neq\left(r_{0}, s_{0}\right)$ and let, for example $r_{0}>r$. Then

$$
\left\langle i_{u}^{r} i_{v}^{s} f_{r s}, i_{u}^{r_{0}} i_{v}^{s_{0}} f_{r_{0} s_{0}}\right\rangle=\left\langle j_{u}^{r_{0}} j_{v}^{s_{0}} i_{u}^{r} i_{v}^{s} f_{r s}, f_{r_{0} s_{0}}\right\rangle .
$$

By (7.9), the right-hand side of the latter formula is zero.

Finally, we prove the existence of representation (7.4). Let us complete the pair $(u, v)$ to form an orthogonal basis $\left(e_{1}, e_{2}, e_{3}, \ldots, e_{n}\right)=\left(u, v, e_{3}, \ldots, e_{n}\right)$ of the space $V$. Any tensor $f \in S^{m}(V)$ can be represented in the form

$$
f=\sum_{|\alpha|=m} \tilde{f}_{\alpha} e^{\alpha}=\sum_{|\alpha|=m} \tilde{f}_{\alpha_{1} \ldots \alpha_{n}} e_{1}^{\alpha_{1}} \ldots e_{n}^{\alpha_{n}}
$$

with some coefficients $\tilde{f}_{\alpha} \in \mathbf{C}$. This can be rewritten as follows:

$$
f=\sum_{r+s \leq m} u^{r} v^{s} \sum_{\alpha_{3}+\ldots+\alpha_{n}=m-r-s} \tilde{f}_{r s \alpha_{3} \ldots \alpha_{n}} e_{3}^{\alpha_{3}} \ldots e_{n}^{\alpha_{n}}=\sum_{r+s \leq m} i_{u}^{r} i_{v}^{s} f_{r s},
$$

where

$$
f_{r s}=\sum_{\alpha_{3}+\ldots+\alpha_{n}=m-r-s} \tilde{f}_{r s \alpha_{3} \ldots \alpha_{n}} e_{3}^{\alpha_{3}} \ldots e_{n}^{\alpha_{n}}
$$

Obviously, $j_{u} f_{r s}=j_{v} f_{r s}=0$.

Lemma 7.6 For a nonzero vector $\xi \in V$, the operator $j_{\xi} i_{\xi}: S^{m} \rightarrow S^{m}$ is the isomorphism whose inverse is given by the formula

$$
\left(j_{\xi} i_{\xi}\right)^{-1}=\frac{1}{|\xi|^{2}} \sum_{k=0}^{m}(-1)^{k}\left(\begin{array}{c}
m+1 \\
k+1
\end{array}\right) \frac{i_{\xi}^{k} j_{\xi}^{k}}{|\xi|^{2 k}}
$$

Proof. By Corollary 7.2,

$$
j_{\xi} i_{\xi}=\frac{|\xi|^{2}}{m+1} E+\frac{m}{m+1} i_{\xi} j_{\xi}
$$

where $E$ is the identity. The operator $i_{\xi} j_{\xi}$ is a nonnegative symmetric operator because it is the product of two operators that are dual to each other. Therefore the operator 
on the right-hand side of the latter formula is a positive symmetric operator, so it is an isomorphism.

To prove the second statement we have just to check that the operator

$$
\frac{j_{\xi} i_{\xi}}{|\xi|^{2}} \sum_{k=0}^{m}(-1)^{k}\left(\begin{array}{c}
m+1 \\
k+1
\end{array}\right) \frac{i_{\xi}^{k} j_{\xi}^{k}}{|\xi|^{2 k}}=\sum_{k=0}^{m}(-1)^{k}\left(\begin{array}{c}
m+1 \\
k+1
\end{array}\right) \frac{j_{\xi} i_{\xi}^{k+1} j_{\xi}^{k}}{|\xi|^{2 k+2}}
$$

coincides with the identity operator. To this end we transpose the factors $j_{\xi}$ and $i_{\xi}^{k+1}$ on the right-hand side of the latter equality using Corollary 7.2

$$
\left(j_{\xi} i_{\xi}^{k+1}\right) j_{\xi}^{k}=\frac{k+1}{m+1}|\xi|^{2} i_{\xi}^{k} j_{\xi}^{k}+\frac{m-k}{m+1} i_{\xi}^{k+1} j_{\xi}^{k+1} .
$$

We substitute the latter expression into (7.10)

$$
\begin{gathered}
\frac{j_{\xi} i_{\xi}}{|\xi|^{2}} \sum_{k=0}^{m}(-1)^{k}\left(\begin{array}{c}
m+1 \\
k+1
\end{array}\right) \frac{i_{\xi}^{k} j_{\xi}^{k}}{|\xi|^{2 k}}= \\
=\sum_{k=0}^{m}(-1)^{k} \frac{k+1}{m+1}\left(\begin{array}{c}
m+1 \\
k+1
\end{array}\right) \frac{i_{\xi}^{k} j_{\xi}^{k}}{|\xi|^{2 k}}+\sum_{k=0}^{m}(-1)^{k} \frac{m-k}{m+1}\left(\begin{array}{c}
m+1 \\
k+1
\end{array}\right) \frac{i_{\xi}^{k+1} j_{\xi}^{k+1}}{|\xi|^{2 k+2}} .
\end{gathered}
$$

The term corresponding to $k=m$ in the second sum can be omitted since $j_{\xi}^{m+1}=0$ on $S^{m}$. Changing the summation index $k:=k-1$ in the second sum and distinguishing the first term of the first sum, we obtain

$$
\begin{gathered}
\frac{j_{\xi} i_{\xi}}{|\xi|^{2}} \sum_{k=0}^{m}(-1)^{k}\left(\begin{array}{c}
m+1 \\
k+1
\end{array}\right) \frac{i_{\xi}^{k} j_{\xi}^{k}}{|\xi|^{2 k}}= \\
=E+\frac{1}{m+1} \sum_{k=1}^{m}(-1)^{k}\left[(k+1)\left(\begin{array}{c}
m+1 \\
k+1
\end{array}\right)-(m-k+1)\left(\begin{array}{c}
m+1 \\
k
\end{array}\right)\right] \frac{i_{\xi}^{k} j_{\xi}^{k}}{|\xi|^{2 k}} .
\end{gathered}
$$

The coefficient in the brackets is equal to zero. We have thus concluded that

$$
\frac{j_{\xi} i_{\xi}}{|\xi|^{2}} \sum_{k=0}^{m}(-1)^{k}\left(\begin{array}{c}
m+1 \\
k+1
\end{array}\right) \frac{i_{\xi}^{k} j_{\xi}^{k}}{|\xi|^{2 k}}=E
$$

The lemma is proved.

\section{Invariant subspaces for the operator $A$}

We return to considering operator (6.6). Recall that the operator is considered for vectors $0 \neq \xi^{\prime} \in V=T_{x}^{*} M$ which are orthogonal to a distinguished unit vector $\nu \in V$.

By Lemma 7.5, there is the orthogonal decomposition $S^{m}=\bigoplus_{r+s \leq m} S_{r s}^{m}$, where $S_{r s}^{m}=$ $S_{r s}^{m}\left(\xi^{\prime}, \nu\right)$ is the subspace of $S^{m}$ consisting of tensors $f$ which can be represented in the form $f=i_{\xi^{\prime}}^{r} i_{\nu}^{s} v$ with $v \in S^{m-r-s}$ satisfying $j_{\xi^{\prime}} v=j_{\nu} v=0$.

Theorem 8.1 For any $0 \leq l \leq m$, the subspace $S_{l}^{m}=\bigoplus_{r+s=l} S_{r s}^{m}$ of $S^{m}$ is invariant under the operator $A\left(\xi^{\prime}\right)$, so we have the orthogonal decomposition $S^{m}=\bigoplus_{l=0}^{m} S_{l}^{m}$ into invariant subspaces. Any tensor $f \in S_{l}^{m}$ can be uniquely represented in the form

$$
f=\sum_{j=0}^{l} i_{\xi^{\prime}}^{l-j} i_{\nu}^{j} v_{j}
$$


with $v_{j} \in S^{m-l}$ satisfying $j_{\xi^{\prime}} v_{j}=j_{\nu} v_{j}=0$. The operator $A\left(\xi^{\prime}\right)$ acts on the space $S_{l}^{m}$ as follows. If $f \in S_{l}^{m}$ is represented in form (8.1), then

$$
A\left(\xi^{\prime}\right) f=\sum_{j=0}^{l} i_{\xi^{\prime}}^{l-j} i_{\nu}^{j} \tilde{v}_{j}
$$

with $\tilde{v}_{j}=\sum_{k=0}^{l} \tilde{a}_{j k}^{(l)} v_{k}$, where

$$
\tilde{a}_{j k}^{(l)}=\frac{j+1}{k+1}\left(i\left|\xi^{\prime}\right|\right)^{j-k} c_{l-k, k}^{k-j}
$$

and

$$
c_{r s}^{t}=\sum_{p=\max (0, t-1)}^{s} \frac{(-1)^{p}}{2^{p+1}}\left(\begin{array}{c}
s+1 \\
p+1
\end{array}\right)\left(\begin{array}{c}
2 p-t+1 \\
p
\end{array}\right) \sum_{q=\max (0,-t)}^{\min (r, p-t+1)} \frac{1}{2^{q}}\left(\begin{array}{l}
r \\
q
\end{array}\right)\left(\begin{array}{c}
p+1 \\
q+t
\end{array}\right) .
$$

The number $c_{r s}^{t}$ is well defined for $r \geq 0, s \geq 0$, and $-r \leq t \leq s+1$.

Corollary 8.2 Given an integer $l \geq 0$, define the $(l+1) \times(l+1)$-matrix $A^{(l)}=\left(a_{j k}^{(l)}\right)_{j, k=0}^{l}$ by

$$
a_{j k}^{(l)}=c_{l-k, k}^{k-j},
$$

where $c_{r s}^{t}$ are given by (8.4). Let $l_{0} \leq+\infty$ be the minimum of such $l$ that $E-A^{(l)}$ is a singular matrix, where $E$ is the unit matrix. Then the operator (7.1) is nondegenerate for any $m<l_{0}$ and for all $\xi^{\prime} \neq 0$, and degenerate for any $m \geq l_{0}$.

Proof of Corollary. The operator $E-A\left(\xi^{\prime}\right): S^{m} \rightarrow S^{m}$ is degenerate if and only if its restriction to $S_{l}^{m}$ is degenerate for some $l \leq m$. The latter happens if and only if the system

$$
\sum_{k=0}^{l}\left(\delta_{j k}-\tilde{a}_{j k}^{(l)}\right) v_{k}=0 \quad(0 \leq j \leq l)
$$

has a nontrivial solution $\left(v_{0}, \ldots, v_{l}\right)$. This is equivalent to degeneracy of the matrix $E-\tilde{A}^{(l)}$, where $\tilde{A}^{(l)}=\left(\tilde{a}_{j k}^{(l)}\right)$.

Comparing (8.3) and (8.5), we see that the matrices $E-\tilde{A}^{(l)}$ and $E-A^{(l)}$ are related by the formula $\delta_{j k}-\tilde{a}_{j k}^{(l)}=\frac{\alpha_{j}}{\alpha_{k}}\left(\delta_{j k}-a_{j k}^{(l)}\right)$, where $\alpha_{j}=\left(i\left|\xi^{\prime}\right|\right)^{-j}(j+1) \neq 0$. This means that the matrix $E-\tilde{A}^{(l)}$ is obtained from $E-A^{(l)}$ by multiplying every $j$-th row by $\alpha_{j}$ and multiplying every $k$-th column by $\left(\alpha_{k}\right)^{-1}$. So, these matrices are degenerate or nondegenerate simultaneously.

Proof of Theorem 8.1. Fix nonnegative integers $r, s$, and $m$ such that $r+s \leq m$. Choose a tensor $v \in S^{m-r-s}$ satisfying

$$
j_{\xi^{\prime}} v=j_{\nu} v=0
$$

and let $f=i_{\xi^{\prime}}^{r} i_{\nu}^{s} v$. Our goal is to represent the tensor $A\left(\xi^{\prime}\right) f$ in the form (8.2) with $l=r+s$.

By (6.6), for $f=i_{\xi^{\prime}}^{r} i_{\nu}^{s} v$

$$
A\left(\xi^{\prime}\right) f=\left.\sum_{k=0}^{m} \frac{(-1)^{k}}{k !}\left(\begin{array}{c}
m+1 \\
k+1
\end{array}\right) \frac{1}{\left(2 i\left|\xi^{\prime}\right|\right)^{k+1}} j_{\nu} \frac{d^{k}}{d \xi_{n}^{k}}\left(i_{\xi}^{k+1} j_{\xi}^{k} i_{\xi^{\prime}}^{r} i_{\nu}^{s} v\right)\right|_{\xi_{n}=i\left|\xi^{\prime}\right|} .
$$


Here $\xi=\xi^{\prime}+\xi_{n} \nu$, so $\left\langle\xi, \xi^{\prime}\right\rangle=\left|\xi^{\prime}\right|^{2}$ and $\langle\xi, \nu\rangle=\xi_{n}$.

First we transpose the factors $j_{\xi}^{k}$ and $i_{\xi^{\prime}}^{r}$ in the expression $i_{\xi}^{k+1} j_{\xi}^{k} i_{\xi^{\prime}}^{r} i_{\nu}^{s} v$. By Lemma 7.1,

$$
\left(j_{\xi}^{k} i_{\xi^{\prime}}^{r}\right) i_{\nu}^{s} v=\sum_{p=\max (0, k-r)}^{\min (k, m-r)} \frac{\left(\begin{array}{c}
m-r \\
p
\end{array}\right)\left(\begin{array}{c}
r \\
k-p
\end{array}\right)}{\left(\begin{array}{c}
m \\
k
\end{array}\right)}\left|\xi^{\prime}\right|^{2(k-p)} i_{\xi^{\prime}}^{p+r-k} j_{\xi}^{p} i_{\nu}^{s} v
$$

Next, we transpose the factors $j_{\xi}^{p}$ and $i_{\nu}^{s}$ in the expression $i_{\xi^{\prime}}^{p+r-k} j_{\xi}^{p} i_{\nu}^{s} v$. By Corollary 7.4

$$
i_{\xi^{\prime}}^{p+r-k}\left(j_{\xi}^{p} i_{\nu}^{s}\right) v=\left\{\begin{array}{l}
0 \quad \text { if } \quad p>s \\
\frac{\left(\begin{array}{c}
s \\
p
\end{array}\right)}{\left(\begin{array}{c}
m-r \\
p
\end{array}\right)} \xi_{n}^{p} i_{\xi^{\prime}}^{p+r-k} i_{\nu}^{s-p} v \quad \text { if } \quad p \leq s .
\end{array}\right.
$$

We substitute the latter expression into (8.8) and apply the operator $i_{\xi}^{k+1}$ to the resultant equation

$$
i_{\xi}^{k+1} j_{\xi}^{k} i_{\xi^{\prime}}^{r} i_{\nu}^{s} v=\sum_{p=\max (0, k-r)}^{\min (k, s)} \frac{\left(\begin{array}{c}
s \\
p
\end{array}\right)\left(\begin{array}{c}
r \\
k-p
\end{array}\right)}{\left(\begin{array}{c}
m \\
k
\end{array}\right)}\left|\xi^{\prime}\right|^{2(k-p)} \xi_{n}^{p} i_{\xi^{\prime}}^{p+r-k} i_{\xi}^{k+1} i_{\nu}^{s-p} v .
$$

Here we have used the equality $\min (k, s, m-r)=\min (k, s)$ that follows from $r+s \leq m$.

Since $i_{\xi}=i_{\xi^{\prime}}+\xi_{n} i_{\nu}$, we have

$$
i_{\xi}^{k+1}=\left(i_{\xi^{\prime}}+\xi_{n} i_{\nu}\right)^{k+1}=\sum_{l=0}^{k+1}\left(\begin{array}{c}
k+1 \\
l
\end{array}\right) \xi_{n}^{l} i_{\xi^{\prime}}^{k-l+1} i_{\nu}^{l} .
$$

We substitute the latter expression into (8.9) and differentiate the resultant equality

$$
\frac{d^{k}}{d \xi_{n}^{k}}\left(i_{\xi}^{k+1} j_{\xi}^{k} i_{\xi^{\prime}}^{r} i_{\nu}^{s} v\right)=\sum_{p=\max (0, k-r)}^{\min (k, s)} \sum_{l=k-p}^{k+1} \frac{\left(\begin{array}{c}
s \\
p
\end{array}\right)\left(\begin{array}{c}
r \\
k-p
\end{array}\right)\left(\begin{array}{c}
k+1 \\
l
\end{array}\right)}{\left(\begin{array}{c}
m \\
k
\end{array}\right)} \frac{(p+l) !}{(p+l-k) !}\left|\xi^{\prime}\right|^{2(k-p)} \xi_{n}^{p+l-k} i_{\xi^{\prime}}^{p+r-l+1} i_{\nu}^{s+l-p} v
$$

We set $\xi_{n}=i\left|\xi^{\prime}\right|$ here and apply the operator $j_{\nu}$ to the resultant formula

$$
\begin{gathered}
\left.j_{\nu} \frac{d^{k}}{d \xi_{n}^{k}}\left(i_{\xi}^{k+1} j_{\xi}^{k} i_{\xi^{\prime}}^{r} i_{\nu}^{s} v\right)\right|_{\xi_{n}=i\left|\xi^{\prime}\right|}= \\
=\sum_{p=\max (0, k-r)}^{\min (k, s)} \sum_{l=k-p}^{k+1} \frac{\left(\begin{array}{c}
s \\
p
\end{array}\right)\left(\begin{array}{c}
r \\
k-p
\end{array}\right)\left(\begin{array}{c}
k+1 \\
l
\end{array}\right)}{\left(\begin{array}{c}
m \\
k
\end{array}\right)} \frac{i^{p+l-k}(p+l) !\left|\xi^{\prime}\right|^{k+l-p}}{(p+l-k) !} j_{\nu} i_{\xi^{\prime}}^{p+r-l+1} i_{\nu}^{s+l-p} v .
\end{gathered}
$$

Next, we transpose the factors $j_{\nu}$ and $i_{\xi^{\prime}}^{p+r-l+1}$ in the expression $j_{\nu} i_{\xi^{\prime}}^{p+r-l+1} i_{\nu}^{s+l-p} v$. By Corollary 7.3 ,

$$
\left(j_{\nu} i_{\xi^{\prime}}^{p+r-l+1}\right) i_{\nu}^{s+l-p} v=\frac{m+l-r-p}{m+1} i_{\xi^{\prime}}^{p+r-l+1} j_{\nu} i_{\nu}^{s+l-p} v
$$

Then, we transpose the factors $j_{\nu}$ and $i_{\nu}^{s+l-p}$ on the right-hand side of (8.11). By Corollary 7.4 ,

$$
i_{\xi^{\prime}}^{p+r-l+1}\left(j_{\nu} i_{\nu}^{s+l-p}\right) v=\frac{s+l-p}{m+l-r-p} i_{\xi^{\prime}}^{p+r-l+1} i_{\nu}^{s+l-p-1} v .
$$


Combining the latter formula with (8.11), we get

$$
j_{\nu} i_{\xi^{\prime}}^{p+r-l+1} i_{\nu}^{s+l-p} v=\frac{s+l-p}{m+1} i_{\xi^{\prime}}^{p+r-l+1} i_{\nu}^{s+l-p-1} v .
$$

Substituting the latter value into (8.10), we obtain

$$
\begin{gathered}
\left.j_{\nu} \frac{d^{k}}{d \xi_{n}^{k}}\left(i_{\xi}^{k+1} j_{\xi}^{k} i_{\xi^{r}}^{r} i_{\nu}^{s} v\right)\right|_{\xi_{n}=i\left|\xi^{\prime}\right|}= \\
=\sum_{p=\max (0, k-r)}^{\min (k, s)} \sum_{l=k-p}^{k+1} \frac{\left(\begin{array}{c}
s \\
p
\end{array}\right)\left(\begin{array}{c}
r \\
k-p
\end{array}\right)\left(\begin{array}{c}
k+1 \\
l
\end{array}\right)}{\left(\begin{array}{c}
m \\
k
\end{array}\right)} \frac{i^{p+l-k}(s+l-p)(p+l) !\left|\xi^{\prime}\right|^{k+l-p}}{(m+1)(p+l-k) !} i_{\xi^{\prime}}^{p+r-l+1} i_{\nu}^{s+l-p-1} v .
\end{gathered}
$$

Finally, we substitute the latter expression into (8.7)

$$
A\left(\xi^{\prime}\right) f=\sum_{k=0}^{m} \sum_{p=\max (0, k-r)}^{\min (k, s)} \sum_{l=k-p}^{k+1} \frac{\left(\begin{array}{c}
s \\
p
\end{array}\right)\left(\begin{array}{c}
r \\
k-p
\end{array}\right)\left(\begin{array}{c}
k+1 \\
l
\end{array}\right)\left(\begin{array}{c}
p+l \\
k
\end{array}\right) i^{p+l-1}(s+l-p)\left|\xi^{\prime}\right|^{l-p-1}}{2^{k+1}(k+1)} i_{\xi^{\prime}}^{p+r-l+1} i_{\nu}^{s+l-p-1} v .
$$

In order to simplify formula (8.12), we remind that the summation indices satisfy the inequalities $l \geq k-p, \quad s \geq p, \quad k \geq p$ which imply $l \geq p-s$. Moreover, the term corresponding to $l=p-s$ is equal to zero because of the presence of the factor $(s+l-p)$. Therefore we can assume that $l \geq p-s+1$, and formula (8.12) can be written in the more precise form:

$$
\begin{gathered}
A\left(\begin{array}{c}
\xi^{\prime} \\
)
\end{array}\right) f= \\
\sum_{k=0}^{m} \sum_{p=\max (0, k-r)}^{\min (k, s)} \sum_{l=\max (p-s+1, k-p)}^{k+1} \frac{\left(\begin{array}{c}
s \\
p
\end{array}\right)\left(\begin{array}{c}
r \\
k-p
\end{array}\right)\left(\begin{array}{c}
k+1 \\
l
\end{array}\right)\left(\begin{array}{c}
p+l \\
k
\end{array}\right) i^{p+l-1}(s+l-p)\left|\xi^{\prime}\right|^{l-p-1}}{2^{k+1}(k+1)} i_{\xi^{\prime}}^{p+r-l+1} i_{\nu}^{s+l-p-1} v .
\end{gathered}
$$

Next, we replace the summation index $l$ with $t=p-l+1$

$$
A\left(\xi^{\prime}\right) f=\sum_{k=0}^{m} \sum_{p=\max (0, k-r)}^{\min (k, s)} \sum_{t=p-k}^{\min (s, 2 p-k+1)} \frac{(-1)^{p}\left(\begin{array}{c}
s \\
p
\end{array}\right)\left(\begin{array}{c}
r \\
k-p
\end{array}\right)\left(\begin{array}{c}
k+1 \\
p-t+1
\end{array}\right)\left(\begin{array}{c}
2 p-t+1 \\
k
\end{array}\right)(s-t+1)\left(i\left|\xi^{\prime}\right|\right)^{-t}}{2^{k+1}(k+1)} i_{\xi^{\prime}}^{r+t} i_{\nu}^{s-t} v .
$$

Since the term $i_{\xi^{\prime}}^{r+t} i_{\nu}^{s-t} v$ in (8.13) is independent of the indices $k$ and $p$, it is quite natural to change the order of summations in (8.13) in such a way that the summation over $t$ would become the most exterior summation. Inequalities $t \geq p-k$ and $p \geq k-r$ imply $t \geq-r$. Thus, the limits for the exterior summation over $t$ are determined by the inequalities $-r \leq t \leq s$. To determine the limits for $k$ and $p$ when $t$ is fixed, we write down the system of inequalities

$$
0 \leq p \leq k, \quad k-r \leq p \leq s, \quad p-k \leq t \leq 2 p-k+1
$$

which is equivalent to the system

$$
0 \leq p, \quad k-r \leq p, \quad \frac{1}{2}(k+t-1) \leq p ; \quad p \leq s, \quad p \leq k, \quad p \leq k+t .
$$

Therefore the limits of summation over $k$ and $p$ are determined by

$$
0 \leq k \leq m ; \quad \max (0, k-r,(k+t-1) / 2) \leq p \leq \min (k, s, k+t) .
$$


Implementing the change, we obtain

$$
A\left(\xi^{\prime}\right)\left(i_{\xi^{\prime}}^{r} i_{\nu}^{s} v\right)=\sum_{t=-r}^{s} \tilde{c}_{r s}^{t}\left(i\left|\xi^{\prime}\right|\right)^{-t} i_{\xi^{\prime}}^{r+t} i_{\nu}^{s-t} v
$$

where

$$
\tilde{c}_{r s}^{t}=(s-t+1) \sum_{k=0}^{m} \frac{1}{2^{k+1}} \sum_{p=\max (0, k-r,(k+t-1) / 2)}^{\min (k, s, k+t)} \frac{(-1)^{p}\left(\begin{array}{c}
s \\
p
\end{array}\right)\left(\begin{array}{c}
r \\
k-p
\end{array}\right)\left(\begin{array}{c}
k+1 \\
p-t+1
\end{array}\right)\left(\begin{array}{c}
2 p-t+1 \\
k
\end{array}\right)}{k+1} .
$$

In particular, formula (8.14) proves the first statement of the theorem.

Next, we change the summation order in (8.15). The index $p$ must satisfy inequalities $0 \leq p \leq s$. Beside this, the inequalities $2 p \geq k+t-1$ and $k \geq p$ imply $p \geq t-1$. So, the summation limits over $p$ are determined by $\max (0, t-1) \leq p \leq s$. In which limits does $k$ vary for a fixed $\mathrm{p}$ ? To answer the question we write down the system

$$
0 \leq k \leq m, \quad k-r \leq p \leq k, \quad(k+t-1) / 2 \leq p \leq k+t
$$

which is equivalent to the following one:

$$
p \leq k, \quad p-t \leq k ; \quad k \leq r+p, \quad k \leq 2 p-t+1 .
$$

Thus, the summation limits over $k$ are determined by

$$
\max (p, p-t) \leq k \leq \min (r+p, 2 p-t+1) .
$$

Implementing the change, we obtain

$$
\tilde{c}_{r s}^{t}=(s-t+1) \sum_{p=\max (0, t-1)}^{s}(-1)^{p}\left(\begin{array}{l}
s \\
p
\end{array}\right) \sum_{k=\max (p, p-t)}^{\min (r+p, 2 p-t+1)} \frac{\left(\begin{array}{c}
r \\
k-p
\end{array}\right)\left(\begin{array}{c}
k+1 \\
p-t+1
\end{array}\right)\left(\begin{array}{c}
2 p-t+1 \\
k
\end{array}\right)}{2^{k+1}(k+1)} .
$$

Then we use the fact that

$$
\frac{\left(\begin{array}{c}
k+1 \\
p-t+1
\end{array}\right)\left(\begin{array}{c}
2 p-t+1 \\
k
\end{array}\right)}{k+1}=\frac{\left(\begin{array}{c}
2 p-t+1 \\
p
\end{array}\right)\left(\begin{array}{c}
p+1 \\
k-p+t
\end{array}\right)}{p+1}
$$

and replace the summation index $k$ with $q=k-p$ to obtain

$$
\tilde{c}_{r s}^{t}=(s-t+1) \sum_{p=\max (0, t-1)}^{s} \frac{(-1)^{p}\left(\begin{array}{l}
s \\
p
\end{array}\right)\left(\begin{array}{c}
2 p-t+1 \\
p
\end{array}\right)}{2^{p+1}(p+1)} \sum_{q=\max (0,-t)}^{\min (r, p-t+1)} \frac{\left(\begin{array}{l}
r \\
q
\end{array}\right)\left(\begin{array}{l}
p+1 \\
q+t
\end{array}\right)}{2^{q}} .
$$

Finally, we transform the formula to the final form

$$
\tilde{c}_{r s}^{t}=\frac{s-t+1}{s+1} \sum_{p=\max (0, t-1)}^{s} \frac{(-1)^{p}}{2^{p+1}}\left(\begin{array}{c}
s+1 \\
p+1
\end{array}\right)\left(\begin{array}{c}
2 p-t+1 \\
p
\end{array}\right) \sum_{q=\max (0,-t)}^{\min (r, p-t+1)} \frac{1}{2^{q}}\left(\begin{array}{l}
r \\
q
\end{array}\right)\left(\begin{array}{l}
p+1 \\
q+t
\end{array}\right)
$$

Let a tensor $f \in S_{l}^{m}$ be represented in the form $f=\sum_{k=0}^{l} i_{\xi^{\prime}}^{l-k} i_{\nu}^{k} v_{k}$ with $v_{k} \in S^{m-l}$ satisfying $j_{\xi^{\prime}} v_{k}=j_{\nu} v_{k}=0$. Setting $v=v_{k}, r=l-k, s=k$ in (8.14), we have

$$
A\left(\xi^{\prime}\right)\left(i_{\xi^{\prime}}^{l-k} i_{\nu}^{k} v_{k}\right)=\sum_{t=k-l}^{k} \tilde{c}_{l-k, k}^{t}\left(i\left|\xi^{\prime}\right|\right)^{-t} i_{\xi^{\prime}}^{l-k+t} i_{\nu}^{k-t} v_{k}=\sum_{j=0}^{l} \tilde{c}_{l-k, k}^{k-j}\left(i\left|\xi^{\prime}\right|\right)^{j-k} i_{\xi^{\prime}}^{l-j} i_{\nu}^{j} v_{k} .
$$


Therefore

$$
A\left(\xi^{\prime}\right) f=\sum_{k=0}^{l} \sum_{j=0}^{l} \tilde{c}_{l-k, k}^{k-j}\left(i\left|\xi^{\prime}\right|\right)^{j-k} i_{\xi^{\prime}}^{l-j} i_{\nu}^{j} v_{k}=\sum_{j=0}^{l} i_{\xi^{\prime}}^{l-j} i_{\nu}^{j}\left(\sum_{k=0}^{l} \tilde{c}_{l-k, k}^{k-j}\left(i\left|\xi^{\prime}\right|\right)^{j-k} v_{k}\right) .
$$

This can be written in the form

$$
A\left(\xi^{\prime}\right) f=\sum_{j=0}^{l} i_{\xi^{\prime}}^{l-j} i_{\nu}^{j} \tilde{v}_{j}
$$

where $\tilde{v}_{j}=\sum_{k=0}^{l} b_{j k}^{l} v_{k}$ and

$$
b_{j k}^{l}=\left(i\left|\xi^{\prime}\right|\right)^{j-k} \tilde{c}_{l-k, k}^{k-j} .
$$

Formulas (8.16)-(8.18) are equivalent to (8.2)-(8.4). This finishes the proof.

\section{Preliminaries on binomial coefficients}

The goal of the section is Lemma 9.3 below. Before proving the lemma, we remind some basic formulas about binomial coefficients. The most of these formulas are known, see for example [4] or [6].

Binomial coefficients are correctly defined by the formula

$$
\left(\begin{array}{l}
x \\
n
\end{array}\right)=\frac{\Gamma(x+1)}{\Gamma(n+1) \Gamma(x-n+1)}
$$

in each of the following two cases: (1) $x \notin\{-1,-2, \ldots\}$ and $n$ is an arbitrary complex number; (2) $n$ is a nonnegative integer and $x$ is an arbitrary complex number. In the latter case the formula

$$
\left(\begin{array}{l}
x \\
n
\end{array}\right)=\frac{x(x-1) \ldots(x-n+1)}{n !}
$$

holds which shows that $\left(\begin{array}{l}x \\ n\end{array}\right)$ is a polynomial of degree $n$ in $x$ with a nonzero leading coefficient. In other words, the family $\left\{\left(\begin{array}{l}x \\ n\end{array}\right) \mid n=0,1, \ldots\right\}$ is the basis of the vector space of polynomials of the variable $x$. The multiplication table in the basis is given by the formula

$$
\left(\begin{array}{l}
x \\
m
\end{array}\right)\left(\begin{array}{l}
x \\
n
\end{array}\right)=\sum_{k=\max (m, n)}^{m+n}\left(\begin{array}{c}
n \\
k-m
\end{array}\right)\left(\begin{array}{l}
k \\
n
\end{array}\right)\left(\begin{array}{l}
x \\
k
\end{array}\right) .
$$

Note that the coefficient

$$
\left(\begin{array}{c}
n \\
k-m
\end{array}\right)\left(\begin{array}{l}
k \\
n
\end{array}\right)=\frac{k !}{(k-m) !(k-n) !(k-m-n) !}
$$

of the formula is symmetric in $(m, n)$. The formula can be easily proved by induction on $n$.

The following formula is an analog of Newton's binomial formula

$$
\left(\begin{array}{c}
x+y \\
n
\end{array}\right)=\sum_{k=0}^{n}\left(\begin{array}{c}
x \\
n-k
\end{array}\right)\left(\begin{array}{l}
y \\
k
\end{array}\right) \text {. }
$$


It is enough to prove the formula for integer $x \geq 0$. Indeed, both parts of the formula are polynomials of $x$. If they coincide for integer values of $x$, then they are equal identically. For integer $x \geq 0$, the formula can be easily proved by induction on $x$. Formula (9.2) has the following corollary:

$$
\sum_{k=0}^{n}\left(\begin{array}{c}
x \\
n-k
\end{array}\right)\left(\begin{array}{c}
-x \\
k
\end{array}\right)=\left\{\begin{array}{lll}
1 & \text { for } & n=0 \\
0 & \text { for } & n>0
\end{array}\right.
$$

The following formula is valid for nonnegative integers $m$ and $n$ :

$$
\sum_{k=0}^{n}(-1)^{k}\left(\begin{array}{l}
n \\
k
\end{array}\right)\left(\begin{array}{c}
x-k \\
m
\end{array}\right)= \begin{cases}0 & \text { for } \quad m<n \\
\left(\begin{array}{c}
x-n \\
m-n
\end{array}\right) & \text { for } m \geq n .\end{cases}
$$

The formula is proved by induction on $n$ on the base of the Pascal equation

$$
\left(\begin{array}{c}
n+1 \\
k
\end{array}\right)=\left(\begin{array}{l}
n \\
k
\end{array}\right)+\left(\begin{array}{c}
n \\
k-1
\end{array}\right) \text { for } k>0 .
$$

Formula (9.4) admits the following generalization: for nonnegative integers $m, n$ and $l$

$$
\sum_{k=0}^{n}(-1)^{k}\left(\begin{array}{l}
k \\
l
\end{array}\right)\left(\begin{array}{l}
n \\
k
\end{array}\right)\left(\begin{array}{c}
x-k \\
m
\end{array}\right)=\left\{\begin{array}{l}
0 \quad \text { for } m+l<n \\
(-1)^{l}\left(\begin{array}{c}
n \\
l
\end{array}\right)\left(\begin{array}{c}
x-n \\
m+l-n
\end{array}\right) \text { for } m+l \geq n .
\end{array}\right.
$$

The latter formula can be reduced to (9.4) with the help of the identity

$$
\left(\begin{array}{l}
k \\
l
\end{array}\right)\left(\begin{array}{l}
n \\
k
\end{array}\right)=\left(\begin{array}{l}
n \\
l
\end{array}\right)\left(\begin{array}{l}
n-l \\
k-l
\end{array}\right) .
$$

Formula (9.4) has one more useful corollary:

$$
\left(\begin{array}{c}
x-y \\
n
\end{array}\right)=\sum_{k=0}^{n}(-1)^{k}\left(\begin{array}{l}
x-k \\
n-k
\end{array}\right)\left(\begin{array}{l}
y \\
k
\end{array}\right) .
$$

Again, it is enough to prove the formula for nonnegative integers $x$ and $y$. In the latter case it can be obtained by setting $n=y$ and $m=x-n$ in (9.4).

Finally, we will need the formula

$$
\sum_{k=0}^{n}(-1)^{k}\left(\begin{array}{l}
x \\
k
\end{array}\right)=(-1)^{n}\left(\begin{array}{c}
x-1 \\
n
\end{array}\right)
$$

which can be obtained by setting $y=-1$ in $(9.2)$ and using the relation $\left(\begin{array}{c}-1 \\ k\end{array}\right)=(-1)^{k}$.

Given an integer $k \geq 0$, we define the linear functional $L_{k}$ on the space of polynomials $f(x)$ by

$$
L_{k}[f(x)]=\sum_{p=0}^{k}(-1)^{p}\left(\begin{array}{l}
k+1 \\
p+1
\end{array}\right) f(p) .
$$

Lemma 9.1 Let a polynomial $f(x)$ satisfy $f(-1)=0$. The degree of $f$ is smaller or equal than $d \geq 1$ if and only if $L_{k}[f]=0$ for $k \geq d$. If such a polynomial is represented in the form $f(x)=\sum_{n=0}^{d} f_{n}\left(\begin{array}{c}x+1 \\ n\end{array}\right)$, then $f_{0}=0$ and $f_{d}=(-1)^{d+1} L_{d-1}[f]$. 
Proof. Represent $f$ as $f(x)=\sum_{n \geq 0} f_{n}\left(\begin{array}{c}x+1 \\ n\end{array}\right)$. The condition $f(-1)=0$ is equivalent to $f_{0}=0$. Therefore

$$
L_{k}[f]=\sum_{n \geq 1} f_{n} L_{k}\left[\left(\begin{array}{c}
x+1 \\
n
\end{array}\right)\right]
$$

On using the equalities

$$
L_{k}\left[\left(\begin{array}{c}
x+1 \\
n
\end{array}\right)\right]=\left\{\begin{array}{c}
0, \quad \text { if } \quad 0<n<k+1 \\
(-1)^{k}, \quad \text { if } \quad n=k+1
\end{array}\right.
$$

which can be easily proved, we obtain

$$
L_{k}[f]=(-1)^{k} f_{k+1} \quad \text { if } \quad f_{k+2}=f_{k+3}=\ldots=0 .
$$

Therefore the condition $f_{d+1}=f_{d+2}=\ldots=0$ is equivalent to $L_{d}[f]=L_{d+1}[f]=\ldots=0$. The lemma is proved.

Let $P$ be the space of polynomials (with real coefficients) of two variables $x$ and $y$. Given an integer $d \geq 1$, we define the subspace $P_{d} \subset P$ as follows: a polynomial $f(x, y)$ belongs to $P_{d}$ if

(1) the degree of $f(x, y)$ with respect to $(x, y)$ is not more than $2 d-1$;

(2) the degree of $f(x, y)$ with respect to $y$ is not more than $d-1$;

(3) the degree of the polynomial $f(x, z-x)$ with respect to $x$ is not more than $d$.

Lemma 9.2 The system of polynomials

$$
\varphi^{(i j)}(x, y)=\left(\begin{array}{c}
x+1 \\
i
\end{array}\right)\left(\begin{array}{c}
x+y \\
j
\end{array}\right) \quad(0 \leq i \leq d, 0 \leq j \leq d-1)
$$

is the basis of the space $P_{d}$.

Proof. It is clear that all $\varphi^{(i j)}$ belong to $P_{d}$. Setting $\psi^{(i j)}(x, z)=\varphi^{(i j)}(x, z-x)$, we obtain the system of polynomials

$$
\psi^{(i j)}(x, z)=\left(\begin{array}{c}
x+1 \\
i
\end{array}\right)\left(\begin{array}{l}
z \\
j
\end{array}\right) \quad(0 \leq i \leq d, 0 \leq j \leq d-1),
$$

which is obviously linearly independent. Therefore system (9.10) is also linearly independent.

Let us show that any $f \in P_{d}$ is a linear combination of system (9.10). Let $g(x, z)=$ $f(x, z-x)$. By the definition of $P_{d}$, the degree of $g(x, z)$ with respect to $x$ is not more than $d$, and the degree of $g(x, z)$ with respect to $z$ is not more than $d-1$. Therefore the representation

$$
g(x, z)=\sum_{i=0}^{d} \sum_{j=0}^{d-1} g_{i j} \psi^{(i j)}(x, z)
$$

exists. It implies

$$
f(x, y)=g(x, x+y)=\sum_{i=0}^{d} \sum_{j=0}^{d-1} g_{i j} \varphi^{(i j)}(x, y) .
$$

The lemma is proved. 
We define the involution $I: P \rightarrow P$ of the space of polynomials of two variables as follows. Every $f \in P$ can be uniquely represented as a finite sum

$$
f(x, y)=\sum_{m, n \geq 0} f_{m n}\left(\begin{array}{c}
x+1 \\
m
\end{array}\right)\left(\begin{array}{l}
y \\
n
\end{array}\right) .
$$

By the definition

$$
(I f)(x, y)=\sum_{m, n \geq 0}(-1)^{m+n} f_{m n}\left(\begin{array}{c}
x+1 \\
m
\end{array}\right)\left(\begin{array}{c}
y \\
n
\end{array}\right) .
$$

Lemma 9.3 (1) The space $P_{d}$ is invariant under the involution $I$.

(2) Let a polynomial $f \in P_{d}$ satisfy $f(-1, y)=0$. Expand it in the basis (9.10)

$$
f(x, y)=\sum_{i=0}^{d} \sum_{j=0}^{d-1} f_{i j} \varphi^{(i j)}(x, y) .
$$

Let $h(x, y)=(I f)(x, z-x)$. The leading coefficient of the representation

$$
h(x, z)=\sum_{k=0}^{d} h_{k}(z)\left(\begin{array}{c}
x+1 \\
k
\end{array}\right)
$$

is expressed by the formula

$$
h_{d}(z)=(-1)^{d} \sum_{j=0}^{d-1} f_{d j} \sum_{p=0}^{j}(-1)^{p}\left(\begin{array}{c}
d-1 \\
j-p
\end{array}\right)\left(\begin{array}{c}
z-d+1 \\
p
\end{array}\right) .
$$

Proof. To prove the first statement, it is enough to check that $I \varphi^{(i j)} \in P_{d}$ for all elements of basis (9.10). To prove the second statement, we have to find the leading coefficient of the decomposition

$$
\chi^{(i j)}(x, z)=\left(I \varphi^{(i j)}\right)(x, z-x)=\sum_{k=0}^{d} \chi_{k}^{(i j)}(z)\left(\begin{array}{c}
x+1 \\
k
\end{array}\right) .
$$

Both statements are obvious in the case $d=1$. Therefore we assume $d \geq 2$ in the proof.

We start the proof of the statement $I \varphi^{(i j)} \in P_{d}$ with considering the case of $i=0$.

$$
\varphi^{(0 j)}(x, y)=\left(\begin{array}{c}
x+y \\
j
\end{array}\right) \quad(0 \leq j \leq d-1)
$$

is a polynomial of degree $j$ in $(x, y)$ admitting the representation

$$
\varphi^{(0 j)}(x, y)=\sum_{0 \leq m+n \leq j} \varphi_{m n}^{(0 j)}\left(\begin{array}{c}
x+1 \\
m
\end{array}\right)\left(\begin{array}{l}
y \\
n
\end{array}\right) .
$$

Therefore

$$
\left(I \varphi^{(0 j)}\right)(x, y)=\sum_{0 \leq m+n \leq j}(-1)^{m+n} \varphi_{m n}^{(0 j)}\left(\begin{array}{c}
x+1 \\
m
\end{array}\right)\left(\begin{array}{c}
y \\
n
\end{array}\right) .
$$


For $j \leq d-1$, each term of the latter sum obviously belongs to $P_{d}$. Moreover, the degree of $\left(I \varphi^{(0 j)}\right)(x, x-z)$ with respect to $x$ is not more than $d-1$. This means, in notations of formula (9.14), that

$$
\chi_{d}^{(0 j)}(z)=0 \quad(0 \leq j \leq d-1) .
$$

From now on, in the proof, we consider the case $i>0$.

First of all we will find the coefficients of the decomposition

$$
\varphi^{(i j)}(x, y)=\sum_{m, n \geq 0} \varphi_{m n}^{(i j)}\left(\begin{array}{c}
x+1 \\
m
\end{array}\right)\left(\begin{array}{c}
y \\
n
\end{array}\right) .
$$

By formula (9.2),

$$
\left(\begin{array}{c}
x+y \\
j
\end{array}\right)=\left(\begin{array}{c}
(x+1)+(y-1) \\
j
\end{array}\right)=\sum_{k=0}^{j}\left(\begin{array}{c}
x+1 \\
j-k
\end{array}\right)\left(\begin{array}{c}
y-1 \\
k
\end{array}\right)=\sum_{k=0}^{j}(-1)^{k}\left(\begin{array}{c}
x+1 \\
j-k
\end{array}\right) \sum_{n=0}^{k}(-1)^{n}\left(\begin{array}{l}
y \\
n
\end{array}\right)
$$

Substituting the latter expression into definition (9.10) of the polynomial $\varphi^{(i j)}$, we obtain

$$
\varphi^{(i j)}(x, y)=\sum_{k=0}^{j} \sum_{n=0}^{k}(-1)^{k+n}\left(\begin{array}{c}
x+1 \\
i
\end{array}\right)\left(\begin{array}{c}
x+1 \\
j-k
\end{array}\right)\left(\begin{array}{c}
y \\
n
\end{array}\right) .
$$

By (9.1),

$$
\left(\begin{array}{c}
x+1 \\
i
\end{array}\right)\left(\begin{array}{c}
x+1 \\
j-k
\end{array}\right)=\sum_{m=\max (i, j-k)}^{i+j-k}\left(\begin{array}{c}
j-k \\
m-i
\end{array}\right)\left(\begin{array}{c}
m \\
j-k
\end{array}\right)\left(\begin{array}{c}
x+1 \\
m
\end{array}\right) .
$$

Substituting this expression into the previous formula, we get

$$
\varphi^{(i j)}(x, y)=\sum_{k=0}^{j} \sum_{n=0}^{k}(-1)^{k+n} \sum_{m=\max (i, j-k)}^{i+j-k}\left(\begin{array}{c}
j-k \\
m-i
\end{array}\right)\left(\begin{array}{c}
m \\
j-k
\end{array}\right)\left(\begin{array}{c}
x+1 \\
m
\end{array}\right)\left(\begin{array}{c}
y \\
n
\end{array}\right) .
$$

After changing the summation order, the formula takes the form

$$
\varphi^{(i j)}(x, y)=\sum_{n=0}^{j}(-1)^{n} \sum_{m=i}^{i+j-n} \varphi_{m n}^{(i j)}\left(\begin{array}{c}
x+1 \\
m
\end{array}\right)\left(\begin{array}{c}
y \\
n
\end{array}\right)
$$

where

$$
\varphi_{m n}^{(i j)}=\sum_{k=\max (n, j-m)}^{i+j-m}(-1)^{k}\left(\begin{array}{c}
j-k \\
m-i
\end{array}\right)\left(\begin{array}{c}
m \\
j-k
\end{array}\right) \quad(0 \leq n \leq j, i \leq m \leq i+j-n) .
$$

We will simplify the latter formula. First we change the summation index as $k:=j-k$

$$
\varphi_{m n}^{(i j)}=(-1)^{j} \sum_{k=m-i}^{\min (m, j-n)}(-1)^{k}\left(\begin{array}{c}
k \\
m-i
\end{array}\right)\left(\begin{array}{c}
m \\
k
\end{array}\right)
$$

Using the equality

$$
\left(\begin{array}{c}
k \\
m-i
\end{array}\right)\left(\begin{array}{c}
m \\
k
\end{array}\right)=\left(\begin{array}{c}
m \\
i
\end{array}\right)\left(\begin{array}{c}
i \\
m-k
\end{array}\right)
$$


we transform the formula to

$$
\varphi_{m n}^{(i j)}=(-1)^{j}\left(\begin{array}{c}
m \\
i
\end{array}\right) \sum_{k=m-i}^{\min (m, j-n)}(-1)^{k}\left(\begin{array}{c}
i \\
m-k
\end{array}\right)=(-1)^{j+m}\left(\begin{array}{c}
m \\
i
\end{array}\right) \sum_{k=\max (0, m+n-j)}^{i}(-1)^{k}\left(\begin{array}{c}
i \\
k
\end{array}\right) .
$$

If $m+n-j \leq 0$, then the sum in the latter formula equals zero. If $m+n-j>0$, we continue our transformations as follows:

$$
\varphi_{m n}^{(i j)}=(-1)^{j+m}\left(\begin{array}{c}
m \\
i
\end{array}\right) \sum_{k=m+n-j}^{i}(-1)^{k}\left(\begin{array}{c}
i \\
i-k
\end{array}\right)=(-1)^{i+j+m}\left(\begin{array}{c}
m \\
i
\end{array}\right) \sum_{k=0}^{i+j-m-n}(-1)^{k}\left(\begin{array}{c}
i \\
k
\end{array}\right) .
$$

We changed again the summation index as $k:=k-m-n+j$ on the last step. Finally, applying (9.8), we obtain

$$
\varphi_{m n}^{(i j)}=(-1)^{n}\left(\begin{array}{c}
m \\
i
\end{array}\right)\left(\begin{array}{c}
i-1 \\
i+j-m-n
\end{array}\right) .
$$

This formula is valid in the case of $m+n-j \leq 0$ too because the right-hand side vanishes in the case.

Substituting the latter value into (9.17), we obtain

$$
\varphi^{(i j)}(x, y)=\sum_{n=0}^{j} \sum_{m=i}^{i+j-n}\left(\begin{array}{c}
m \\
i
\end{array}\right)\left(\begin{array}{c}
i-1 \\
i+j-m-n
\end{array}\right)\left(\begin{array}{c}
x+1 \\
m
\end{array}\right)\left(\begin{array}{c}
y \\
n
\end{array}\right) .
$$

Observe that the summation limits in (9.18) are determined by the summand. Indeed, the factor $\left(\begin{array}{c}m \\ i\end{array}\right)$ is not zero only if $i \leq m$, and the factor $\left(\begin{array}{c}i-1 \\ i+j-m-n\end{array}\right)$ is not zero only if $m \leq i+j-n$. Inequalities $i \leq m \leq i+j-n$ imply $n \leq j$. Therefore formula (9.18) can be simplified to the following one:

$$
\varphi^{(i j)}(x, y)=\sum_{m, n \geq 0}\left(\begin{array}{c}
m \\
i
\end{array}\right)\left(\begin{array}{c}
i-1 \\
i+j-m-n
\end{array}\right)\left(\begin{array}{c}
x+1 \\
m
\end{array}\right)\left(\begin{array}{c}
y \\
n
\end{array}\right) .
$$

We apply the involution $I$ to (9.19)

$$
\left(I \varphi^{(i j)}\right)(x, y)=\sum_{m, n \geq 0}(-1)^{m+n}\left(\begin{array}{c}
m \\
i
\end{array}\right)\left(\begin{array}{c}
i-1 \\
i+j-m-n
\end{array}\right)\left(\begin{array}{c}
x+1 \\
m
\end{array}\right)\left(\begin{array}{c}
y \\
n
\end{array}\right) .
$$

We have to prove that $I \varphi^{(i j)} \in P_{d}$ for $0<i \leq d, j \leq d-1$. It is clear from (9.20) that $\left(I \varphi^{(i j)}\right)(x, y)$ is a polynomial of degree $\leq i+j \leq 2 d-1$ with respect to $(x, y)$ since the factor $\left(\begin{array}{c}i-1 \\ i+j-m-n\end{array}\right)$ can be nonzero only for $m+n \leq i+j$. Second, inequalities $i \leq m$ and $m+n \leq i+j$ imply $n \leq j$, i.e., $\left(I \varphi^{(i j)}\right)(x, y)$ is a polynomial of degree $\leq j \leq d-1$ with respect to $y$. It remains to prove that

$$
\chi^{(i j)}(x, z)=\left(I \varphi^{(i j)}\right)(x, z-x)=\sum_{m, n \geq 0}(-1)^{m+n}\left(\begin{array}{c}
m \\
i
\end{array}\right)\left(\begin{array}{c}
i-1 \\
i+j-m-n
\end{array}\right)\left(\begin{array}{c}
x+1 \\
m
\end{array}\right)\left(\begin{array}{c}
z-x \\
n
\end{array}\right)
$$

is a polynomial of degree $\leq d$ with respect to $x$. 
Observe that $\chi^{(i j)}(-1, z)=0$ for $i>0$ because only positive values of $m$ participate in sum (9.21). Thus, Lemma 9.1 can be applied. By the lemma, the statement $\operatorname{deg}_{x} \chi^{(i j)} \leq d$ is equivalent to the following one:

$$
L_{k}\left[\chi^{(i j)}(x, z)\right]=0 \quad \text { for } \quad k \geq d .
$$

Hereafter, the functional $L_{k}$ acts with respect to the variable $x$ while $z$ is considered as a parameter. The latter equality should be valid identically in $z$.

As we know, $\chi^{(i j)}(x, z)$ is a polynomial of degree $\leq i+j$, i.e., $L_{k}\left[\chi^{(i j)}(x, z)\right]=0$ for $k \geq i+j$. So, it is enough to consider the case of $d \leq k<i+j$. The question is thus reduced to the statement

$$
L_{k}\left[\chi^{(i j)}(x, z)\right]=0 \quad \text { for } \quad d \leq k<i+j, 0<i \leq d, 0 \leq j \leq d-1 .
$$

The case of $k=d-1$ is also of some interest for us because we want to find the leading coefficient of decomposition (9.14).

Let

$$
1 \leq d-1 \leq k<i+j, \quad 0<i \leq d, \quad 0 \leq j \leq d-1 .
$$

By definition (9.9) of the functional $L_{k}$ and formula (9.21),

$$
L_{k}\left[\chi^{(i j)}\right]=\sum_{p=0}^{k}(-1)^{p}\left(\begin{array}{l}
k+1 \\
p+1
\end{array}\right) \sum_{m, n \geq 0}(-1)^{m+n}\left(\begin{array}{c}
m \\
i
\end{array}\right)\left(\begin{array}{c}
i-1 \\
i+j-m-n
\end{array}\right)\left(\begin{array}{c}
p+1 \\
m
\end{array}\right)\left(\begin{array}{c}
z-p \\
n
\end{array}\right) .
$$

The term of the sum can be nonzero only if $i \leq m \leq p+1$. Therefore the summation with respect to $p$ can be restricted to the limits $i-1 \leq p \leq k$, and the summation with respect to $m$ can be restricted to the limits $i \leq m \leq p+1$. We rewrite the formula in the more precise form

$$
L_{k}\left[\chi^{(i j)}\right]=\sum_{p=i-1}^{k}(-1)^{p}\left(\begin{array}{l}
k+1 \\
p+1
\end{array}\right) \sum_{m=i}^{p+1} \sum_{n \geq 0}(-1)^{m+n}\left(\begin{array}{c}
m \\
i
\end{array}\right)\left(\begin{array}{c}
i-1 \\
i+j-m-n
\end{array}\right)\left(\begin{array}{c}
p+1 \\
m
\end{array}\right)\left(\begin{array}{c}
z-p \\
n
\end{array}\right) .
$$

Changing the summation order, we have

$$
L_{k}\left[\chi^{(i j)}\right]=\sum_{m=i}^{k+1} \sum_{n \geq 0}(-1)^{m+n}\left(\begin{array}{c}
m \\
i
\end{array}\right)\left(\begin{array}{c}
i-1 \\
i+j-m-n
\end{array}\right) A_{m n}^{k}(z)
$$

where

$$
A_{m n}^{k}(z)=\sum_{p=m-1}^{k}(-1)^{p}\left(\begin{array}{c}
k+1 \\
p+1
\end{array}\right)\left(\begin{array}{c}
p+1 \\
m
\end{array}\right)\left(\begin{array}{c}
z-p \\
n
\end{array}\right) .
$$

Formula (9.25) can be simplified. Indeed, using the equality

$$
\left(\begin{array}{c}
k+1 \\
p+1
\end{array}\right)\left(\begin{array}{c}
p+1 \\
m
\end{array}\right)=\left(\begin{array}{c}
k+1 \\
m
\end{array}\right)\left(\begin{array}{c}
k-m+1 \\
p-m+1
\end{array}\right)
$$

we transform the formula to the form

$$
A_{m n}^{k}(z)=\left(\begin{array}{c}
k+1 \\
m
\end{array}\right) \sum_{p=m-1}^{k}(-1)^{p}\left(\begin{array}{c}
k-m+1 \\
p-m+1
\end{array}\right)\left(\begin{array}{c}
z-p \\
n
\end{array}\right) .
$$


Then, changing the summation index as $p:=p+m-1$, we have

$$
A_{m n}^{k}(z)=(-1)^{m+1}\left(\begin{array}{c}
k+1 \\
m
\end{array}\right) \sum_{p=0}^{k-m+1}(-1)^{p}\left(\begin{array}{c}
k-m+1 \\
p
\end{array}\right)\left(\begin{array}{c}
z-m+1-p \\
n
\end{array}\right) .
$$

The sum on the right-hand side of this formula has the same structure as the left-hand side of (9.4). Applying the latter formula, we obtain

$$
A_{m n}^{k}(z)=\left\{\begin{array}{l}
0 \quad \text { for } n<k-m+1 \\
(-1)^{m+1}\left(\begin{array}{c}
k+1 \\
m
\end{array}\right)\left(\begin{array}{c}
z-k \\
m+n-k-1
\end{array}\right)
\end{array} \text { for } n \geq k-m+1 .\right.
$$

We substitute the latter value into (9.24)

$$
L_{k}\left[\chi^{(i j)}\right]=\sum_{m=i}^{k+1} \sum_{n \geq k-m+1}(-1)^{n+1}\left(\begin{array}{c}
m \\
i
\end{array}\right)\left(\begin{array}{c}
i-1 \\
i+j-m-n
\end{array}\right)\left(\begin{array}{c}
k+1 \\
m
\end{array}\right)\left(\begin{array}{c}
z-k \\
m+n-k-1
\end{array}\right)
$$

and replace the summation index $n$ with $r=m+n$

$$
L_{k}\left[\chi^{(i j)}\right]=-\sum_{m=i}^{k+1} \sum_{r \geq k+1}(-1)^{m+r}\left(\begin{array}{c}
m \\
i
\end{array}\right)\left(\begin{array}{c}
i-1 \\
i+j-r
\end{array}\right)\left(\begin{array}{c}
k+1 \\
m
\end{array}\right)\left(\begin{array}{c}
z-k \\
r-k-1
\end{array}\right) .
$$

The summation in the inner sum can be restricted to the limits $k+1 \leq r \leq i+j$ because the factor $\left(\begin{array}{c}i-1 \\ i+j-r\end{array}\right)$ equals zero for $r>i+j$. Changing the order of summation, we rewrite the latter formula in the form

$$
L_{k}\left[\chi^{(i j)}\right]=-b_{k}^{i} \sum_{r=k+1}^{i+j}(-1)^{r}\left(\begin{array}{c}
i-1 \\
i+j-r
\end{array}\right)\left(\begin{array}{c}
z-k \\
r-k-1
\end{array}\right),
$$

where

$$
b_{k}^{i}=\sum_{m=i}^{k+1}(-1)^{m}\left(\begin{array}{c}
m \\
i
\end{array}\right)\left(\begin{array}{c}
k+1 \\
m
\end{array}\right) .
$$

We transform formula (9.27). Using the equality

$$
\left(\begin{array}{c}
m \\
i
\end{array}\right)\left(\begin{array}{c}
k+1 \\
m
\end{array}\right)=\left(\begin{array}{c}
k+1 \\
i
\end{array}\right)\left(\begin{array}{c}
k-i+1 \\
m-i
\end{array}\right)
$$

the formula takes the form

$$
b_{k}^{i}=\left(\begin{array}{c}
k+1 \\
i
\end{array}\right) \sum_{m=i}^{k+1}(-1)^{m}\left(\begin{array}{c}
k-i+1 \\
m-i
\end{array}\right) .
$$

Changing the summation index as $m:=m+i$, we see that

$$
b_{k}^{i}=(-1)^{i}\left(\begin{array}{c}
k+1 \\
i
\end{array}\right) \sum_{m=0}^{k-i+1}(-1)^{m}\left(\begin{array}{c}
k-i+1 \\
m
\end{array}\right)=\left\{\begin{array}{l}
0, \text { if } i<k+1, \\
(-1)^{k+1}, \quad \text { if } i=k+1 .
\end{array}\right.
$$

Together with the latter relation, formula (9.26) gives that

$$
L_{k}\left[\chi^{(i j)}\right]=0 \quad \text { for } \quad i<k+1
$$


and

$$
L_{k}\left[\chi^{(k+1, j)}\right]=(-1)^{k} \sum_{r=k+1}^{j+k+1}(-1)^{r}\left(\begin{array}{c}
k \\
j+k-r+1
\end{array}\right)\left(\begin{array}{c}
z-k \\
r-k-1
\end{array}\right) .
$$

Recall that the values of $i, j$ and $k$ are assumed to satisfy the inequalities (9.23). If $k \geq d$, then $i \leq d<k+1$, and formula (9.28) gives (9.22). This proves the first statement of the lemma.

If $k=d-1$, then (9.28) and (9.29) give

$$
L_{d-1}\left[\chi^{(i j)}\right]=0 \quad \text { for } \quad 0<i<d
$$

and

$$
L_{d-1}\left[\chi^{(d j)}\right]=(-1)^{d+1} \sum_{r=d}^{j+d}(-1)^{r}\left(\begin{array}{c}
d-1 \\
d+j-r
\end{array}\right)\left(\begin{array}{c}
z-d+1 \\
r-d
\end{array}\right) .
$$

Replacing the summation index $r$ with $m=r-d$ in the latter formula, we have

$$
L_{d-1}\left[\chi^{(d j)}\right]=-\sum_{m=0}^{j}(-1)^{m}\left(\begin{array}{c}
d-1 \\
j-m
\end{array}\right)\left(\begin{array}{c}
z-d+1 \\
m
\end{array}\right) .
$$

We can now finish the proof of the second statement of the lemma. If a polynomial $f(x, y)$ satisfies $f(-1, y)=0$, then the coefficients $f_{0 j}$ in representation (9.11) are equal to zero. In decomposition (9.12) of the polynomial $h(x, z)=(I f)(x, z-x)$, the coefficient $h_{0}(z)$ is also equal to zero as one can easily see. Therefore Lemma 9.1 can be applied to $h(x, z)$. By the lemma,

$$
h_{d}(z)=(-1)^{d+1} L_{d-1}[h(x, z)] .
$$

On the other hand, applying the involution $I$ to equality (9.11), we have

$$
(I f)(x, y)=\sum_{i=1}^{d} \sum_{j=0}^{d-1} f_{i j}\left(I \varphi^{(i j)}\right)(x, y) .
$$

Set $y=x-z$ here

$$
h(x, z)=\sum_{i=1}^{d} \sum_{j=0}^{d-1} f_{i j} \chi^{(i j)}(x, z) .
$$

Apply the operator $L_{d-1}$ to the latter equality

$$
L_{d-1}[h]=\sum_{i=1}^{d} \sum_{j=0}^{d-1} f_{i j} L_{d-1}\left[\chi^{(i j)}\right]
$$

and use (9.32) and (9.33)

$$
L_{d-1}[h]=-\sum_{j=0}^{d-1} f_{d j} \sum_{m=0}^{j}(-1)^{m}\left(\begin{array}{c}
d-1 \\
j-m
\end{array}\right)\left(\begin{array}{c}
z-d+1 \\
m
\end{array}\right) .
$$

Comparing the latter formula with (9.32), we obtain (9.13).

The lemma is proved. 


\section{The matrix $E-A^{(l)}$ is nonsingular}

By Corollary 8.2, to finish the proof of Theorem 1.1, we have to demonstrate that the matrix $E-A^{(l)}$ is nonsingular for any $l$. We remind that the matrix $A^{(l)}=\left(a_{j k}^{(l)}\right)_{j, k=0}^{l}$ is defined by

$$
a_{j k}^{(l)}=c_{l-k, k}^{k-j}
$$

where $c_{r s}^{t}$ are given by (8.4).

Theorem 10.1 The numbers

$$
\lambda_{j}^{(l)}=\frac{1}{2^{l+1}}\left[2 \sum_{p=0}^{j}\left(\begin{array}{l}
l \\
p
\end{array}\right)-\left(\begin{array}{l}
l \\
j
\end{array}\right)\right] \quad(0 \leq j \leq l)
$$

are eigenvalues of the matrix $A^{(l)}$.

Note that $\lambda_{j}^{(l)} \neq \lambda_{k}^{(l)}$ for $j \neq k, 0<\lambda_{j}^{(l)}<1$, and $\lambda_{j}^{(l)}=1-\lambda_{l-j}^{(l)}$. Thus, the spectrum $\Lambda^{(l)}=\left\{\lambda_{j}^{(l)} \mid 0 \leq j \leq l\right\}$ of the matrix $A^{(l)}$ consists of $l+1$ different eigenvalues, lies in the interval $(0,1)$, and is symmetric with respect to the point $\lambda=1 / 2$. Therefore the matrix $A^{(l)}$ is diagonalizable and the matrix $E-A^{(l)}$ has the same spectrum $\Lambda^{(l)}$. In particular, $E-A^{(l)}$ is a nonsingular matrix.

Define the matrix $U^{(l)}=\left(u_{j k}\right)_{j, k=0}^{l}$ by the equality

$$
u_{j k}=\left(\begin{array}{c}
k+1 \\
j+1
\end{array}\right)
$$

Note that $U^{(l)}$ is an upper-triangular matrix with units on the diagonal and the inverse matrix $\left(U^{(l)}\right)^{-1}=\left(v_{j k}\right)$ is given by

$$
v_{j k}=(-1)^{j+k}\left(\begin{array}{c}
k+1 \\
j+1
\end{array}\right) .
$$

Theorem 10.1 is the obvious corollary of the following statement

Lemma 10.2 The product $\left(U^{(l)}\right)^{-1} A^{(l)} U^{(l)}$ is a low-triangular matrix with the numbers $\lambda_{0}^{(l)}, \ldots, \lambda_{l}^{(l)}$ on the diagonal.

Written in components, the statement of Lemma 10.2 looks as follows:

$$
\sum_{r, s=0}^{l} v_{j r} a_{r s}^{(l)} u_{s k}=\left\{\begin{array}{l}
0 \text { for } j<k \\
\lambda_{k}^{(l)} \text { for } j=k
\end{array}\right.
$$

We substitute values (10.1), (10.3), and (10.4) into the latter formula and replace the summation index $r$ with $t=s-r$ to obtain

$$
\sum_{s=0}^{k}(-1)^{s}\left(\begin{array}{c}
k+1 \\
s+1
\end{array}\right) \sum_{t=s-l}^{s-j}(-1)^{t}\left(\begin{array}{c}
s-t+1 \\
j+1
\end{array}\right) c_{l-s, s}^{t}=\left\{\begin{array}{l}
0 \text { for } j<k, \\
(-1)^{k} \lambda_{k}^{(l)} \text { for } j=k .
\end{array}\right.
$$


Comparing the latter formula with definition (9.9) of the functional $L_{k}$, we see that the statement of Lemma 10.2 is equivalent to the following one:

$$
L_{k}\left[g_{j}^{(l)}(s)\right]=\left\{\begin{array}{l}
0 \quad \text { for } 1 \leq j \leq k \\
(-1)^{k} \lambda_{k}^{(l)} \text { for } j=k+1
\end{array}\right.
$$

where

$$
g_{j}^{(l)}(s)=\sum_{t=s-l}^{s-j+1}(-1)^{t}\left(\begin{array}{c}
s-t+1 \\
j
\end{array}\right) c_{l-s, s}^{t} .
$$

Taking the convention $c_{r s}^{t}=0$ for $t<-r$ and $t>s+1$, the latter formula can be written in the simpler form

$$
g_{j}^{(l)}(s)=\sum_{t}(-1)^{t}\left(\begin{array}{c}
s-t+1 \\
j
\end{array}\right) c_{l-s, s}^{t},
$$

where the summation is performed over all integers $t$.

Comparing (10.5) with Lemma 9.1, we see that Lemma 10.2 follows from the next statement.

Lemma 10.3 Defined by (10.6), the function $g_{j}^{(l)}(s)$ is a polynomial of degree $\leq j$ in $s$ for any $0 \leq j \leq l+1$. The polynomial satisfies $g_{j}^{(l)}(-1)=0$. In particular, $g_{0}^{(l)}(s) \equiv 0$. The leading coefficient of the decomposition

$$
g_{j}^{(l)}(s)=\sum_{n=0}^{j} g_{j n}^{(l)}\left(\begin{array}{c}
s+1 \\
n
\end{array}\right)
$$

is

$$
g_{j j}^{(l)}=\lambda_{j-1}^{(l)}
$$

using the convention $\lambda_{-1}^{(l)}=0$.

Remark. The statement " $g_{j}^{(l)}(s)$ is a polynomial in s" is not trivial since the number of nonzero terms in sums (10.6) and (8.4) goes to infinity as $s$ does. The simplest example of such a phenomenon is presented by the formula $2^{s}=\sum_{t \geq 0}\left(\begin{array}{l}s \\ t\end{array}\right)$. Each term of the sum depends polynomially on $s$ but the sum is not a polynomial.

We will reduce Lemma 10.3 to the following statement.

Lemma 10.4 For $0 \leq j \leq l+1$, the function

$$
f_{j}^{(l)}(s)=\sum_{t}(-1)^{t}\left(\begin{array}{l}
t \\
j
\end{array}\right) c_{l-s, s}^{t}
$$

is a polynomial of degree $\leq j$ in s satisfying $f_{j}^{(l)}(-1)=0$. In particular, $f_{0}^{(l)}(s) \equiv 0$. The leading coefficient of the decomposition

$$
f_{j}^{(l)}(s)=\sum_{n=0}^{j} f_{j n}^{(l)}\left(\begin{array}{c}
s+1 \\
n
\end{array}\right)
$$

is given by

$$
f_{j j}^{(l)}=-\sum_{p=0}^{j-1}(-1)^{p}\left(\begin{array}{c}
j \\
p+1
\end{array}\right) \lambda_{p}^{(l)} .
$$


Proof of Lemma 10.3. We will express $g_{j}^{(l)}(s)$ through the functions $f_{k}^{(l)}(s)(0 \leq$ $k \leq l+1)$. To this end we write using (9.7)

$$
\left(\begin{array}{c}
s-t+1 \\
j
\end{array}\right)=\sum_{k=0}^{j}(-1)^{k}\left(\begin{array}{c}
s-k+1 \\
j-k
\end{array}\right)\left(\begin{array}{l}
t \\
k
\end{array}\right) .
$$

Substituting this value into (10.6) and comparing the result with (10.9), we obtain

$$
g_{j}^{(l)}(s)=\sum_{k=0}^{j}(-1)^{k}\left(\begin{array}{c}
s-k+1 \\
j-k
\end{array}\right) f_{k}^{(l)}(s) .
$$

Assuming the validity of Lemma 10.4, we see that every term of sum (10.12) is a polynomial of degree $\leq j$ in $s$ vanishing at $s=-1$. Therefore the same is true for $g_{j}^{(l)}(s)$. This proves the first statement of Lemma 10.3. It remains to calculate the leading coefficient of the decomposition (10.7).

Deleting all terms of degree $<j$, we write (10.12) as follows:

$$
g_{j j}^{(l)}\left(\begin{array}{c}
s+1 \\
j
\end{array}\right)=\sum_{k=0}^{j}(-1)^{k} f_{k k}^{(l)}\left(\begin{array}{c}
s+1 \\
j-k
\end{array}\right)\left(\begin{array}{c}
s+1 \\
k
\end{array}\right)+\ldots
$$

Here and in the next formula, the dots stand for a polynomial of degree $<j$ in $s$. By (9.1),

$$
\left(\begin{array}{c}
s+1 \\
j-k
\end{array}\right)\left(\begin{array}{c}
s+1 \\
k
\end{array}\right)=\left(\begin{array}{l}
j \\
k
\end{array}\right)\left(\begin{array}{c}
s+1 \\
j
\end{array}\right)+\ldots
$$

Substituting this value into the previous formula, we have

$$
g_{j j}^{(l)}=\sum_{k=0}^{j}(-1)^{k}\left(\begin{array}{l}
j \\
k
\end{array}\right) f_{k k}^{(l)} .
$$

Insert the value (10.11) for $f_{k k}^{(l)}$ into the latter equality

$$
g_{j j}^{(l)}=-\sum_{k=1}^{j}(-1)^{k}\left(\begin{array}{l}
j \\
k
\end{array}\right) \sum_{p=0}^{k-1}(-1)^{p}\left(\begin{array}{c}
k \\
p+1
\end{array}\right) \lambda_{p}^{(l)} .
$$

After changing the summation order, this formula takes the form

$$
g_{j j}^{(l)}=\sum_{p=0}^{j-1} B_{p}^{j} \lambda_{p}^{(l)}
$$

where

$$
B_{p}^{j}=(-1)^{p+1} \sum_{k=p+1}^{j}(-1)^{k}\left(\begin{array}{l}
j \\
k
\end{array}\right)\left(\begin{array}{c}
k \\
p+1
\end{array}\right) .
$$

The last formula can be simplified. First, with the help of the equality

$$
\left(\begin{array}{l}
j \\
k
\end{array}\right)\left(\begin{array}{c}
k \\
p+1
\end{array}\right)=\left(\begin{array}{c}
j \\
p+1
\end{array}\right)\left(\begin{array}{c}
j-p-1 \\
j-k
\end{array}\right),
$$


we transform the formula to the following one:

$$
B_{p}^{j}=(-1)^{p+1}\left(\begin{array}{c}
j \\
p+1
\end{array}\right) \sum_{k=p+1}^{j}(-1)^{k}\left(\begin{array}{c}
j-p-1 \\
j-k
\end{array}\right) .
$$

Then we replace the summation index $k$ with $r=j-k$

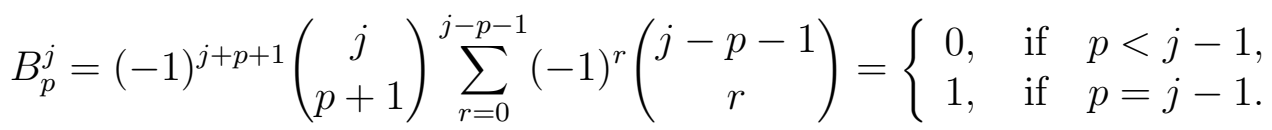

Substituting this value into (10.13), we arrive to the equality $g_{j j}^{(l)}=\lambda_{j-1}^{(l)}$. This finishes the proof of Lemma 10.3.

The rest of the section is devoted to the proof of Lemma 10.4.

Substituting value (8.4) for $c_{l-s, s}^{t}$ into (10.9), we obtain

$$
f_{j}^{(l)}(s)=\sum_{p, q \geq 0} \frac{(-1)^{p+q}}{2^{p+q+1}}\left(\begin{array}{c}
s+1 \\
p+1
\end{array}\right)\left(\begin{array}{c}
l-s \\
q
\end{array}\right) F^{(j)}(p, q),
$$

where

$$
F^{(j)}(p, q)=(-1)^{q} \sum_{t}(-1)^{t}\left(\begin{array}{l}
t \\
j
\end{array}\right)\left(\begin{array}{c}
p+1 \\
q+t
\end{array}\right)\left(\begin{array}{c}
2 p-t+1 \\
p
\end{array}\right) .
$$

The idea of the proof is to separate variables $p$ and $q$ in the function $F^{(j)}(p, q)$, i.e., to represent the function in the form $F^{(j)}(p, q)=\sum_{k} G_{k}(p) H_{k}(q)$. After such a separation, the right-hand side of (10.14) will be factorized to a product of two sums, over $p$ and $q$ independently, which can be easily calculated.

We transform formula (10.15). We replace the summation index $t$ with $k=t+q$ and use the equality

$$
\left(\begin{array}{c}
k-q \\
j
\end{array}\right)=\sum_{i=0}^{j}\left(\begin{array}{c}
-q \\
j-i
\end{array}\right)\left(\begin{array}{l}
k \\
i
\end{array}\right)
$$

that follows from (9.2). We obtain

$$
F^{(j)}(p, q)=\sum_{i=0}^{j}\left(\begin{array}{c}
-q \\
j-i
\end{array}\right) \sum_{k}(-1)^{k}\left(\begin{array}{c}
k \\
i
\end{array}\right)\left(\begin{array}{c}
p+1 \\
k
\end{array}\right)\left(\begin{array}{c}
2 p+q-k+1 \\
p
\end{array}\right) .
$$

The inner sum in (10.16) can be calculated using (9.6):

$$
\sum_{k}(-1)^{k}\left(\begin{array}{c}
k \\
i
\end{array}\right)\left(\begin{array}{c}
p+1 \\
k
\end{array}\right)\left(\begin{array}{c}
2 p+q-k+1 \\
p
\end{array}\right)=\left\{\begin{array}{l}
0 \quad \text { for } i=0, \\
(-1)^{i}\left(\begin{array}{c}
p+1 \\
i
\end{array}\right)\left(\begin{array}{c}
p+q \\
i-1
\end{array}\right) \text { for } \quad i>0 .
\end{array}\right.
$$

This means, in particular, that $F^{0}(p, q) \equiv 0$ and, consequently, $f^{(0)}(s) \equiv 0$ as stated in Lemma 10.4. Therefore we will consider only the case of $j>0$ in what follows. Substituting value (10.17) into (10.16), we obtain

$$
F^{(j)}(p, q)=\sum_{i=1}^{j}(-1)^{i}\left(\begin{array}{c}
p+1 \\
i
\end{array}\right)\left(\begin{array}{c}
-q \\
j-i
\end{array}\right)\left(\begin{array}{c}
p+q \\
i-1
\end{array}\right)
$$


It is clear from $(10.18)$ that $F^{(j)}(p, q)$ is a polynomial in $(p, q)$ and satisfies

$$
F^{(j)}(-1, q)=0 \text {. }
$$

The degree of $F^{(j)}(p, q)$ with respect to $(p, q)$ is equal to $2 j-1$, its degree with respect to $q$ is not larger than $j-1$, and the degree of the polynomial $F^{(j)}(p, l-p)$ with respect to $p$ is not larger than $j$. This means that $F^{(j)}(p, q)$ belongs to the space $P_{j}$ introduced before Lemma 9.2 (where $x, y, z$ and $d$ are replaced with $p, q, l$ and $j$ respectively).

Represent the polynomial $F^{(j)}(p, q)$ as

$$
F^{(j)}(p, q)=\sum_{m, n \geq 0} \tilde{F}_{m n}^{(j)}\left(\begin{array}{c}
p+1 \\
m
\end{array}\right)\left(\begin{array}{c}
q \\
n
\end{array}\right)
$$

substitute this expression into (10.14), and write the result in the form

$$
f_{j}^{(l)}(s)=\sum_{m, n \geq 0} \tilde{F}_{m n}^{(j)} A_{m}(s) B_{n}(l-s)
$$

where

$$
A_{m}(s)=\sum_{p \geq 0} \frac{(-1)^{p}}{2^{p+1}}\left(\begin{array}{c}
s+1 \\
p+1
\end{array}\right)\left(\begin{array}{c}
p+1 \\
m
\end{array}\right)
$$

and

$$
B_{n}(l-s)=\sum_{q \geq 0} \frac{(-1)^{q}}{2^{q}}\left(\begin{array}{c}
l-s \\
q
\end{array}\right)\left(\begin{array}{c}
q \\
n
\end{array}\right) .
$$

Formulas (10.22) and (10.23) are equivalent to

$$
A_{m}(s)=\frac{(-1)^{m+1}}{2^{s+1}}\left(\begin{array}{c}
s+1 \\
m
\end{array}\right)
$$

and

$$
B_{n}(l-s)=\frac{(-1)^{n}}{2^{l-s}}\left(\begin{array}{c}
l-s \\
n
\end{array}\right)
$$

respectively. We will present the proof only of the first of these formulas because the second one is proved in a similar way. Changing the summation index in (10.22), we write the formula as follows:

$$
A_{m}(s)=-\sum_{p \geq 0} \frac{(-1)^{p}}{2^{p}}\left(\begin{array}{c}
s+1 \\
p
\end{array}\right)\left(\begin{array}{c}
p \\
m
\end{array}\right)
$$

In the larrer formula, $m$ is a nonnegative integer and $s$ is considered as a complex variable.

Consider the function $u(x)=(1-x / 2)^{s+1}$ of the complex argument $x$ normalized by the condition $u(0)=1$. It is a holomorphic function in the disc $|x|<2$ with the Tailor series

$$
(1-x / 2)^{s+1}=\sum_{p \geq 0} \frac{(-1)^{p}}{2^{p}}\left(\begin{array}{c}
s+1 \\
p
\end{array}\right) x^{p} .
$$

Applying the operator $\frac{1}{m !} \frac{d^{m}}{d x^{m}}$ to the latter equality we get

$$
\frac{(-1)^{m}}{2^{m}}\left(\begin{array}{c}
s+1 \\
m
\end{array}\right)(1-x / 2)^{s-m+1}=\sum_{p \geq 0} \frac{(-1)^{p}}{2^{p}}\left(\begin{array}{c}
s+1 \\
p
\end{array}\right)\left(\begin{array}{c}
p \\
m
\end{array}\right) x^{p-m} .
$$


Setting $x=1$, obtain

$$
\sum_{p \geq 0} \frac{(-1)^{p}}{2^{p}}\left(\begin{array}{c}
s+1 \\
p
\end{array}\right)\left(\begin{array}{c}
p \\
m
\end{array}\right)=\frac{(-1)^{m}}{2^{s+1}}\left(\begin{array}{c}
s+1 \\
m
\end{array}\right) .
$$

Together with (10.26), the latter formula gives (10.24).

Substituting values (10.24) and (10.25) into (10.21), we obtain

$$
f_{j}^{(l)}(s)=-\frac{1}{2^{l+1}} \sum_{m, n \geq 0}(-1)^{m+n} \tilde{F}_{m n}^{(j)}\left(\begin{array}{c}
s+1 \\
m
\end{array}\right)\left(\begin{array}{c}
l-s \\
n
\end{array}\right) .
$$

Comparing formulas (10.20) and (10.27), we see that

$$
f_{j}^{(l)}(s)=-\frac{1}{2^{l+1}}\left(I F^{(j)}\right)(s, l-s),
$$

where $I$ is the involution of the space of polynomials of the variables $p$ and $q$ defined in the previous section. By Lemma 9.3, the polynomial $\left(I F^{(j)}\right)(p, q)$ belongs to the space $P_{j}$. Now, formula (10.28) shows that $f_{j}^{(l)}(s)$ is a polynomial of degree $\leq j$ in $s$. This proves the first statement of Lemma 10.4.

It remains to calculate the leading coefficient of the polynomial $f_{j}^{(l)}(s)$. To this end, according to the second statement of Lemma 9.3, we have to find the expansion of the polynomial $F^{(j)}(p, q)$ in the basis

$$
\varphi^{(\alpha \beta)}(p, q)=\left(\begin{array}{c}
p+1 \\
\alpha
\end{array}\right)\left(\begin{array}{c}
p+q \\
\beta
\end{array}\right) \quad(0 \leq \alpha \leq j, 0 \leq \beta \leq j-1)
$$

of the space $P_{j}$. In the expansion

$$
F^{(j)}(p, q)=\sum_{\alpha=0}^{j} \sum_{\beta=0}^{j-1} F_{\alpha \beta}^{(j)} \varphi^{(\alpha \beta)}(p, q)
$$

we are interested only in coefficients $F_{\alpha \beta}^{(j)}$ with $\alpha=j$.

Using (9.2), we can write

$$
\left(\begin{array}{c}
-q \\
j-i
\end{array}\right)=\left(\begin{array}{c}
(p+1)+(-p-q-1) \\
j-i
\end{array}\right)=\sum_{\gamma=0}^{j-i}\left(\begin{array}{c}
p+1 \\
\gamma
\end{array}\right)\left(\begin{array}{c}
-p-q-1 \\
j-i-\gamma
\end{array}\right) .
$$

Replacing the second factor on the right-hand side of (10.18) with the latter value, we obtain

$$
F^{(j)}(p, q)=\sum_{i=1}^{j} \sum_{\gamma=0}^{j-i}(-1)^{i}\left(\begin{array}{c}
p+1 \\
i
\end{array}\right)\left(\begin{array}{c}
p+1 \\
\gamma
\end{array}\right)\left(\begin{array}{c}
-p-q-1 \\
j-i-\gamma
\end{array}\right)\left(\begin{array}{c}
p+q \\
i-1
\end{array}\right) .
$$

Every term of the latter sum is the product of a polynomial of degree $i+\gamma \leq j$ of $p$ and a polynomial of $p+q$. We are interested only in those terms for which $i+\gamma=j$. Thus, we can write

$$
F^{(j)}(p, q)=\sum_{i=1}^{j}(-1)^{i}\left(\begin{array}{c}
p+1 \\
i
\end{array}\right)\left(\begin{array}{c}
p+1 \\
j-i
\end{array}\right)\left(\begin{array}{c}
p+q \\
i-1
\end{array}\right)+\ldots
$$

where the dots stand for a linear combination of those $\varphi^{(\alpha \beta)}(p, q)$ for which $\alpha<j$. 
By (9.1),

$$
\left(\begin{array}{c}
p+1 \\
i
\end{array}\right)\left(\begin{array}{c}
p+1 \\
j-i
\end{array}\right)=\left(\begin{array}{c}
j \\
i
\end{array}\right)\left(\begin{array}{c}
p+1 \\
j
\end{array}\right)+\ldots,
$$

where the dots stand for a polynomial of degree $<j$ in $p$. Substituting this expression into (10.31), we have

$$
F^{(j)}(p, q)=\sum_{i=1}^{j}(-1)^{i}\left(\begin{array}{c}
j \\
i
\end{array}\right)\left(\begin{array}{c}
p+1 \\
j
\end{array}\right)\left(\begin{array}{c}
p+q \\
i-1
\end{array}\right)+\ldots
$$

Replacing the summation index $i$ with $\beta=i-1$ and comparing the resultant formula with definition $(10.29)$ of $\varphi^{(\alpha \beta)}(p, q)$, we see that

$$
F^{(j)}(p, q)=-\sum_{\beta=0}^{j-1}(-1)^{\beta}\left(\begin{array}{c}
j \\
\beta+1
\end{array}\right) \varphi^{(j \beta)}(p, q)+\ldots,
$$

where the dots stand for a linear combination of those $\varphi^{(\alpha \beta)}(p, q)$ for which $\alpha<j$. We have thus proved that

$$
F_{j \beta}^{(j)}=(-1)^{\beta+1}\left(\begin{array}{c}
j \\
\beta+1
\end{array}\right)
$$

We conclude from (10.32) with the help of Lemma 9.3, that the leading coefficient of the representation

$$
\left(I F^{(j)}\right)(s, l-s)=\sum_{k=0}^{j} h_{k}(l)\left(\begin{array}{c}
s+1 \\
k
\end{array}\right)
$$

is

$$
h_{j}(l)=(-1)^{j+1} \sum_{\beta=0}^{j-1}(-1)^{\beta}\left(\begin{array}{c}
j \\
\beta+1
\end{array}\right) \sum_{\gamma=0}^{\beta}(-1)^{\gamma}\left(\begin{array}{c}
j-1 \\
\beta-\gamma
\end{array}\right)\left(\begin{array}{c}
l-j+1 \\
\gamma
\end{array}\right) .
$$

Comparing this formula with (10.28), we obtain

$$
f_{j j}^{(l)}=\frac{(-1)^{j}}{2^{l+1}} \sum_{\beta=0}^{j-1}(-1)^{\beta}\left(\begin{array}{c}
j \\
\beta+1
\end{array}\right) \sum_{\gamma=0}^{\beta}(-1)^{\gamma}\left(\begin{array}{c}
j-1 \\
\beta-\gamma
\end{array}\right)\left(\begin{array}{c}
l-j+1 \\
\gamma
\end{array}\right) .
$$

We recall that $f_{j j}^{(l)}$ is the leading coefficient of representation (10.10).

It remains to prove equivalence of formulas (10.11) and (10.34). Let us temporary denote the right-hand side of $(10.11)$ by $\tilde{f}_{j j}^{(l)}$, i.e.,

$$
\tilde{f}_{j j}^{(l)}=-\sum_{\beta=0}^{j-1}(-1)^{\beta}\left(\begin{array}{c}
j \\
\beta+1
\end{array}\right) \lambda_{\beta}^{(l)} .
$$

Substituting the value (10.2) for $\lambda_{\beta}^{(l)}$ into the latter formula, we conclude

$$
\tilde{f}_{j j}^{(l)}=-\frac{1}{2^{l+1}} \sum_{\beta=0}^{j-1}(-1)^{\beta}\left(\begin{array}{c}
j \\
\beta+1
\end{array}\right)\left[2 \sum_{p=0}^{\beta}\left(\begin{array}{l}
l \\
p
\end{array}\right)-\left(\begin{array}{l}
l \\
\beta
\end{array}\right)\right] .
$$

We have to prove that $f_{j j}^{(l)}=\tilde{f}_{j j}^{(l)}$. To this end we will transform both formulas (10.34) and (10.35) to get representations for $f_{j j}^{(l)}$ and $\tilde{f}_{j j}^{(l)}$ as linear combinations of the polynomials 
$\left(\begin{array}{l}l \\ k\end{array}\right)$ of the variable $l$, and then we will prove that these two linear combinations have the same coefficients.

We start with transforming formula (10.35)

$$
\tilde{f}_{j j}^{(l)}=-\frac{1}{2^{l}} \sum_{\beta=0}^{j-1}(-1)^{\beta}\left(\begin{array}{c}
j \\
\beta+1
\end{array}\right) \sum_{p=0}^{\beta}\left(\begin{array}{l}
l \\
p
\end{array}\right)+\frac{1}{2^{l+1}} \sum_{\beta=0}^{j-1}(-1)^{\beta}\left(\begin{array}{c}
j \\
\beta+1
\end{array}\right)\left(\begin{array}{l}
l \\
\beta
\end{array}\right) .
$$

We change the summation order in the first term on the right-hand side and replace the summation index $\beta$ with $p$ in the second term

$$
\tilde{f}_{j j}^{(l)}=-\frac{1}{2^{l}} \sum_{p=0}^{j-1}\left(\begin{array}{l}
l \\
p
\end{array}\right) \sum_{\beta=p}^{j-1}(-1)^{\beta}\left(\begin{array}{c}
j \\
\beta+1
\end{array}\right)+\frac{1}{2^{l+1}} \sum_{p=0}^{j-1}(-1)^{p}\left(\begin{array}{c}
j \\
p+1
\end{array}\right)\left(\begin{array}{l}
l \\
p
\end{array}\right) .
$$

The inner sum of the first term can be easily calculated using (9.8):

$$
\sum_{\beta=p}^{j-1}(-1)^{\beta}\left(\begin{array}{c}
j \\
\beta+1
\end{array}\right)=-\sum_{\beta=p+1}^{j}(-1)^{\beta}\left(\begin{array}{c}
j \\
j-\beta
\end{array}\right)=(-1)^{j+1} \sum_{\beta=0}^{j-p-1}(-1)^{\beta}\left(\begin{array}{l}
j \\
\beta
\end{array}\right)=(-1)^{p}\left(\begin{array}{c}
j-1 \\
p
\end{array}\right) .
$$

Substitute the latter value into the previous formula

$$
\tilde{f}_{j j}^{(l)}=-\frac{1}{2^{l}} \sum_{p=0}^{j-1}(-1)^{p}\left(\begin{array}{c}
j-1 \\
p
\end{array}\right)\left(\begin{array}{l}
l \\
p
\end{array}\right)+\frac{1}{2^{l+1}} \sum_{p=0}^{j-1}(-1)^{p}\left(\begin{array}{c}
j \\
p+1
\end{array}\right)\left(\begin{array}{l}
l \\
p
\end{array}\right) .
$$

Uniting two sums on the right-hand side and using the Pascal relation $\left(\begin{array}{c}j \\ p+1\end{array}\right)=\left(\begin{array}{c}j-1 \\ p\end{array}\right)+\left(\begin{array}{c}j-1 \\ p+1\end{array}\right)$, we obtain

$$
\tilde{f}_{j j}^{(l)}=\frac{1}{2^{l+1}} \sum_{p=0}^{j-1}(-1)^{p}\left[\left(\begin{array}{c}
j-1 \\
p+1
\end{array}\right)-\left(\begin{array}{c}
j-1 \\
p
\end{array}\right)\right]\left(\begin{array}{l}
l \\
p
\end{array}\right) .
$$

Now, we transform formula (10.34). First we write using (9.2)

$$
\left(\begin{array}{c}
l-j+1 \\
\gamma
\end{array}\right)=\sum_{p=0}^{\gamma}\left(\begin{array}{l}
1-j \\
\gamma-p
\end{array}\right)\left(\begin{array}{l}
l \\
p
\end{array}\right)
$$

Insert this expression into (10.34) and change the summation order in the resultant sum

$$
f_{j j}^{(l)}=\frac{(-1)^{j}}{2^{l+1}} \sum_{p=0}^{j-1} \sum_{\beta=p}^{j-1}(-1)^{\beta}\left(\begin{array}{c}
j \\
\beta+1
\end{array}\right) \sum_{\gamma=p}^{\beta}(-1)^{\gamma}\left(\begin{array}{l}
j-1 \\
\beta-\gamma
\end{array}\right)\left(\begin{array}{l}
1-j \\
\gamma-p
\end{array}\right)\left(\begin{array}{l}
l \\
p
\end{array}\right) .
$$

Replacing the summation indices $\beta$ and $\gamma$ with $i=\beta-p$ and $k=\gamma-p$ respectively, we finally get

$$
f_{j j}^{(l)}=\frac{(-1)^{j}}{2^{l+1}} \sum_{p=0}^{j-1}\left[\sum_{i=0}^{j-p-1}(-1)^{i}\left(\begin{array}{c}
j \\
i+p+1
\end{array}\right) \sum_{k=0}^{i}(-1)^{k}\left(\begin{array}{c}
j-1 \\
i-k
\end{array}\right)\left(\begin{array}{c}
1-j \\
k
\end{array}\right)\right]\left(\begin{array}{l}
l \\
p
\end{array}\right) .
$$

Comparing (10.36) and (10.37), we see that the statement $f_{j j}^{(l)}=\tilde{f}_{j j}^{(l)}$ is equivalent to the system

$$
(-1)^{j+p} \sum_{i=0}^{j-p-1}(-1)^{i}\left(\begin{array}{c}
j \\
i+p+1
\end{array}\right) \sum_{k=0}^{i}(-1)^{k}\left(\begin{array}{c}
j-1 \\
i-k
\end{array}\right)\left(\begin{array}{c}
1-j \\
k
\end{array}\right)=\left(\begin{array}{c}
j-1 \\
p+1
\end{array}\right)-\left(\begin{array}{c}
j-1 \\
p
\end{array}\right)
$$


for $0 \leq p \leq j-1$. In order to simplify the formula, we slightly change notations as follows. First replace the variable $j$ with $m=j-1$, and then replace $p$ with $q=m-p$. In such the way obtain

$$
(-1)^{q} \sum_{i=0}^{q}(-1)^{i}\left(\begin{array}{c}
m+1 \\
q-i
\end{array}\right) \sum_{k=0}^{i}(-1)^{k}\left(\begin{array}{c}
m \\
i-k
\end{array}\right)\left(\begin{array}{c}
-m \\
k
\end{array}\right)=\left(\begin{array}{c}
m \\
q
\end{array}\right)-\left(\begin{array}{c}
m \\
q-1
\end{array}\right) \quad \text { for } \quad 0 \leq q \leq m .
$$

The next transformation is used to simplify the right-hand side of (10.38). Choose $p$ satisfying $0 \leq p \leq m$ and take the sum of equations (10.38) over $q$ in the limits $0 \leq q \leq p$. In such the way we obtain the equivalent system

$$
\sum_{q=0}^{p}(-1)^{q} \sum_{i=0}^{q}(-1)^{i}\left(\begin{array}{c}
m+1 \\
q-i
\end{array}\right) \sum_{k=0}^{i}(-1)^{k}\left(\begin{array}{c}
m \\
i-k
\end{array}\right)\left(\begin{array}{c}
-m \\
k
\end{array}\right)=\left(\begin{array}{c}
m \\
p
\end{array}\right) \quad \text { for } \quad 0 \leq p \leq m .
$$

Next, we change the summation order on the left-hand side of the latter formula

$$
\sum_{k=0}^{p}(-1)^{k}\left(\begin{array}{c}
-m \\
k
\end{array}\right) \sum_{i=k}^{p}(-1)^{i}\left(\begin{array}{c}
m \\
i-k
\end{array}\right) \sum_{q=i}^{p}(-1)^{q}\left(\begin{array}{c}
m+1 \\
q-i
\end{array}\right)=\left(\begin{array}{c}
m \\
p
\end{array}\right) \quad \text { for } \quad 0 \leq p \leq m .
$$

The inner sum can be easily calculated on the base of (9.8):

$$
\sum_{q=i}^{p}(-1)^{k}\left(\begin{array}{c}
m+1 \\
q-i
\end{array}\right)=(-1)^{i} \sum_{q=0}^{p-i}(-1)^{q}\left(\begin{array}{c}
m+1 \\
q
\end{array}\right)=(-1)^{p}\left(\begin{array}{c}
m \\
p-i
\end{array}\right) .
$$

Substitute this value into (10.39) to obtain

$$
(-1)^{p} \sum_{k=0}^{p}(-1)^{k}\left(\begin{array}{c}
-m \\
k
\end{array}\right) \sum_{i=k}^{p}(-1)^{i}\left(\begin{array}{c}
m \\
i-k
\end{array}\right)\left(\begin{array}{c}
m \\
p-i
\end{array}\right)=\left(\begin{array}{c}
m \\
p
\end{array}\right) \quad \text { for } \quad 0 \leq p \leq m .
$$

Change the summation order

$$
(-1)^{p} \sum_{i=0}^{p}(-1)^{i}\left(\begin{array}{c}
m \\
p-i
\end{array}\right) \sum_{k=0}^{i}(-1)^{k}\left(\begin{array}{c}
m \\
i-k
\end{array}\right)\left(\begin{array}{c}
-m \\
k
\end{array}\right)=\left(\begin{array}{c}
m \\
p
\end{array}\right) \quad \text { for } \quad 0 \leq p \leq m
$$

replace the summation index $k$ with $j=i-k$

$$
(-1)^{p} \sum_{i=0}^{p}\left(\begin{array}{c}
m \\
p-i
\end{array}\right) \sum_{j=0}^{i}(-1)^{j}\left(\begin{array}{c}
m \\
j
\end{array}\right)\left(\begin{array}{c}
-m \\
i-j
\end{array}\right)=\left(\begin{array}{c}
m \\
p
\end{array}\right) \quad \text { for } \quad 0 \leq p \leq m,
$$

and change again the summation order to obtain

$$
(-1)^{p} \sum_{j=0}^{p}(-1)^{j}\left(\begin{array}{c}
m \\
j
\end{array}\right) \sum_{i=j}^{p}\left(\begin{array}{c}
m \\
p-i
\end{array}\right)\left(\begin{array}{c}
-m \\
i-j
\end{array}\right)=\left(\begin{array}{c}
m \\
p
\end{array}\right) \quad \text { for } \quad 0 \leq p \leq m .
$$

We emphasize that the systems (10.38) and (10.40) are equivalent, i.e., system (10.40) is still equivalent to the equality $f_{j j}^{(l)}=\tilde{f}_{j j}^{(l)}$ for $j=m+1$. At a first glance there does not seem to be any progress in going from (10.38) to (10.40) because these systems seem to have almost the same structure. However, there is the following crucial difference between these systems. All summands of the inner sum in (10.38) are positive (take the 
sign $(-1)^{k}$ of the factor $\left(\begin{array}{c}-m \\ k\end{array}\right)$ into account). On the other hand, the summands of the inner sum in (10.40) have alternating signs and they cancel each other, as is shown in the next paragraph.

We calculate the inner sum of (10.40). To this end we replace the summation index $i$ with $q=i-j$ and use (9.3)

$$
\sum_{i=j}^{p}\left(\begin{array}{c}
m \\
p-i
\end{array}\right)\left(\begin{array}{c}
-m \\
i-j
\end{array}\right)=\sum_{q=0}^{p-j}\left(\begin{array}{c}
m \\
p-j-q
\end{array}\right)\left(\begin{array}{c}
-m \\
q
\end{array}\right)=\left\{\begin{array}{lll}
1 & \text { for } & j=p \\
0 & \text { for } & j<p
\end{array}\right.
$$

Substituting the latter value into (10.40), we arrive to the identity

$$
\left(\begin{array}{c}
m \\
p
\end{array}\right)=\left(\begin{array}{c}
m \\
p
\end{array}\right)
$$

This proves validity of (10.40).

Lemma 10.4 is proved.

\section{References}

[1] Chappa E. On a characterization of the kernel of the geodesic X-ray transform. Transactions $A M S$, to appear.

[2] Chazarain J. and Piriou A. Introduction to the Theory of Linear Partial Differential Equations. Studies in Mathematics and its applications. V. 14. North-Holland Publishing Company, 1982.

[3] Dairbekov N. Deformation boundary rigidity for SGM-manifolds and integral geometry problems for nontrapping manifolds, MPI-Preprint Nr. 04-26, Bonn, 2004, 14 p.

[4] Graham R., Knut D., Patashik O. Concrete Mathematics. Addison — Wesley Publishing Co., Reading, MA, 1989.

[5] Pestov L. Well-Posedness Questions of the Ray Tomography Problems. [Russian]. Siberian Science Press, Novosibirsk, 2003.

[6] Prudnikov A., Brychkov Y., Marichev O. Integrals and Series. Vol. 1. Elementary Functions. New York a.o., London and Breach Publ., 1986.

[7] Sharafutdinov V. Integral Geometry of Tensor Fields. VSP, Utrecht, the Netherlands, 1994.

[8] Sharafutdinov V. Finiteness theorem for the ray transform on a Riemannian manifold. Inverse Problems, 11, 1039-1050, 1995.

[9] Sharafutdinov V. Geometric symbol calculus for pseudodifferential operators. I. Siberian Advances in Math., 15, no. 3, 2005.

[10] Sharafutdinov V. and Uhlmann G. On deformation boundary rigidity and spectral rigidity of Riemannian surfaces with no focal points. J. of Differential Geometry, 56, 93-110, 2000. 
[11] Stefanov P. and Uhlmann G. Stability estimates for the X-ray transform of tensor fields and boundary rigidity. Duke Math. J., to appear. 